\title{
Microscopic structure of a heavily irradiated material
}

\author{
P. M. Derlet ${ }^{1, *}$ and S. L. Dudarev ${ }^{2, \dagger}$ \\ ${ }^{1}$ Condensed Matter Theory Group, Paul Scherrer Institut, CH-5232 Villigen PSI, Switzerland \\ ${ }^{2}$ UK Atomic Energy Authority, Culham Centre for Fusion Energy, Oxfordshire OX14 3DB, United Kingdom
}

(Received 23 December 2019; accepted 31 January 2020; published 24 February 2020)

\begin{abstract}
It has been long hypothesized that the structure of a material bombarded by energetic particles might approach a certain asymptotic steady state in the limit of high exposure to irradiation. There is still no definitive verdict regarding the validity of this hypothesis or the conditions where it applies. To clarify this, we explore a highly simplified model for microstructural evolution that retains full atomic detail of the underlying crystal structure and involves random events of generation and relaxation of defects. We explore the dynamics of evolution of the model in the limit $T=0$, where the defect and dislocation microstructure is driven purely by the spatially fluctuating stress field accumulating as a result of stochastic generation of point defects. Using body-centred cubic iron and tungsten as examples, we show that their microstructure exhibits a structural transition and then approaches a limiting asymptotic state at doses of order $O(0.1)$ and $O(1)$ canonical defects per atom, respectively, and analyze the microscopic and macroscopic parameters characterizing both the transition and the asymptotic microstructural state.
\end{abstract}

DOI: 10.1103/PhysRevMaterials.4.023605

\section{INTRODUCTION}

A variety of applications involve exposing materials to intense fluxes of energetic atomic or subatomic particles, producing significant changes in microstructure. These applications include high-energy accelerators [1], advanced fission or future fusion reactors [2], high-energy ion beam processing of materials [3], immobilization of radioactive waste [4], and surface modification of materials by ion beams [5]. Effects of exposure to energetic particles appear particularly significant in the limit where the density of defects produced in the material by irradiation is high [6].

An accepted measure of exposure to irradiation is a dimensionless positive definite parameter called displacement per atom (DPA) [7]. The notion of atomic displacements, introduced by Kinchin and Pease [8] and quantified by Norgett, Robinson, and Torrens [9], refers to a DPA parameter $\phi$, the derivative of which with respect to time $\dot{\phi}=d \phi / d t$ is proportional to the average rate of production of pairs of vacancy and self-interstitial atom (SIA) defects per atomic site per unit time. Whereas in the low-dose limit $\phi \ll 1$, in the absence of recombination or other kinetic effects, the number of defects produced in a material per atomic site varies linearly with $\phi$, a still outstanding question critical to applications is how the microstructure of a material changes, in comparison with the initial microstructure, in the limit where $\phi \sim 1$ or $\phi \gg 1$.

Somewhat surprisingly, exploring the limit $\phi \gg 1$ by a direct experimental observation involving the use of a high energy ion beam, is relatively routine [10,11]. High levels of exposure exceeding 200 DPA have been achieved using

\footnotetext{
*Peter.Derlet@psi.ch

†Sergei.Dudarev@ukaea.uk
}

neutron irradiation in commercial steels [12]. However, interpreting the data proves challenging, let alone developing a predictive model for the microstructure developing in the high exposure limit.

Remarkable progress in elucidating the fundamental origin of structural changes occurring in materials under irradiation has been achieved over the past three decades using molecular dynamics simulations $[7,13-16]$. Simulations primarily explore the production of defects by impacts of energetic particle in the bulk of perfect crystals $[17,18]$ or near surfaces $[19,20]$. Following the pioneering studies by Samaras et al. [21,22], who analyzed how defects were produced by energetic collision events in nanocrystalline materials, extensive computer simulations have been performed exploring the effect of vacancy clusters $[23,24]$, dislocations $[25,26]$, dislocation loops [27], and grain boundaries [28] on defect production and accumulation in materials where the initial microstructure was different from the ideal periodic crystal lattice. Direct experimental observations show that the characteristic timescales of evolution of microstructure under irradiation depend strongly on the type of microstructure already present in the material. For example, finely dispersed defects appear to slow down the evolution considerably more significantly than defects coalesced into a large-scale dislocation network [29]. Similarly, short-range fluctuations of the local atomic environment in a concentrated solid solution appear to have a profound effect on the evolution of microstructure [30,31].

Particularly valuable information about the defect and dislocation microstructure of a material exposed to a high amount of irradiation can be derived from simulations involving repeated impacts of energetic particles [30,32]. Simulations show, in qualitative agreement with experimental observations [33], that the accumulation of defects gradually slows down and possibly saturates in the $\phi \sim 1$ limit. However, a fully quantitative statistical exploration of the transition to 


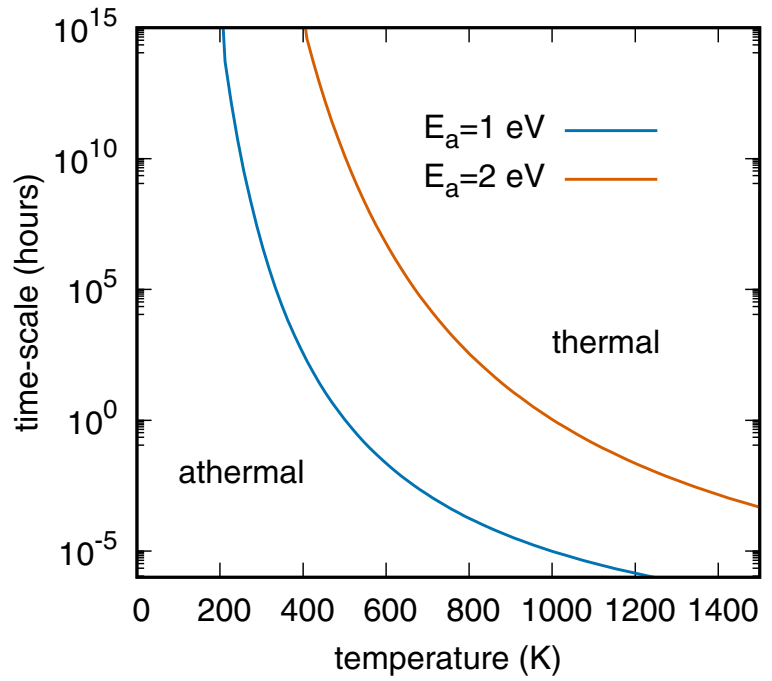

FIG. 1. Sketch of the relaxation time-temperature diagram, plotted for $\omega_{0}=4 \times 10^{13} \mathrm{~s}^{-1}$ and for two values of the effective activation energy $E_{a}$. Microstructure remains stationary at relatively low temperatures and short timescales. Thermally activated dynamic evolution occurs in the high temperature and long timescale asymptotic limit.

saturation and the asymptotic $\phi \gg 1$ limit requires relatively extensive computational effort, and involves simulating a large number of dynamic collision cascade events. The relatively slow cumulative evolution of microstructure as a function of $\phi$ appears to occur in several stages, from the initial linear regime to the eventual asymptotic saturation. The identification of these stages in simulations of dynamic cascade events is impeded by data fluctuations, resulting from a combination of factors. Those include the size of the simulation cell that is often comparable with the spatial scale of a dynamic cascade event, the still less well controlled high-energy part of the simulations sensitive to the assumed rate of energy losses, the uncertainty associated with the small impact parameter interaction, the cut-off range of the interaction potential [34] and possibly even quantum-mechanical nonadiabatic effects $[18,35,36]$.

To examine the fundamental physical content of the problem of exposure to high dose, and to select a suitable simulation algorithm, it is convenient to refer to the relaxation time-temperature diagram shown in Fig. 1. Thermally activated evolution of microstructure in the great majority of cases follows the Arrhenius law [37]. For a given temperature $T$, this law enables defining a characteristic timescale $\tau$ of microstructural evolution as

$$
\tau^{-1} \sim \omega_{0} \exp \left(-E_{a} / k_{B} T\right),
$$

where $\omega_{0}$ is the attempt frequency, comparable to the Debye frequency of the material, and $E_{a}$ is the activation energy for the dominant low-barrier mode of evolution. Defect and dislocation microstructure remains stationary on the timescale shorter than $\tau$.

The magnitude of $\tau$ can be macroscopic. For example the microstructure of pure iron exposed to high-dose ion irradiation evolves over a period of approximately five months at room temperature [38]. The recovery of dislocation loops in aluminium at room temperature occurs on the timescale of 230 years [39]. No significant dynamics is observed in tungsten irradiated to 1.6 DPA on the timescale of an hour [40] at temperatures up to $800^{\circ} \mathrm{C}$. Irradiated zirconium [41] does not visibly evolve at temperatures below $300^{\circ} \mathrm{C}$. The microstructure of irradiated steels remains stationary on the timescale of a week [42] at $T \leqslant 330{ }^{\circ} \mathrm{C}$.

Treating defect and dislocation microstructure as a dynamic entity evolving by thermal activation is only necessary in the limit $t \gg \tau$. If the rate $\dot{\phi}$ of generation of defects by irradiation is higher than $\tau^{-1}$, then microstructural evolution is driven by factors other than thermal activation. Experimental observations show that cases where microstructural evolution occurring under irradiation is not thermally activated, are common [33].

Among the computational methods developed for modeling evolution driven by thermal activation, kinetic Monte Carlo (kMC) methods [43,44] have provided fundamental insights into the dynamics of accumulation of radiation damage. $\mathrm{kMC}$ approaches are particularly successful in the small dose limit $\phi \ll 1$, where the density of defects is low and where simulations match experimental observations very well [45-47]. Applying kMC simulations to dense defect and dislocation microstructures observed in the $\phi \geqslant 1$ limit, and including the effects of linear and nonlinear elastic interaction between defects and dislocations in the kMC framework has so far proved challenging [48-52].

Can the high-dose limit be explored by a direct atomistic simulation? Studies involving applications of molecular dynamics to modeling high entropy alloys, exposed to a relatively high irradiation dose, were carried out by Koch et al. [53] and Granberg et al. [30]. The simulations, involving random initiations of many hundreds of cascade events in the same simulation cell, have enabled reaching the level of exposure close to $\phi \sim 0.7$. In principle, this approach can be extended to the high-dose limit, however the extensive computational effort associated with direct atomistic simulations of cascades make the above studies relatively unique.

It is appropriate to recall the fundamental meaning of the exposure parameter $\phi$. This parameter was introduced in Refs. [8,9] as an estimate for the average rate of production of point defects in a material exposed to a flux of energetic particles. Modern computer simulations aim to predict how these defects are produced by directly modeling high energy collision events $[7,16,54]$. While the simulations successfully mimic the microscopic dynamics of atomic collisions, the instabilities invariably associated with solutions of nonlinear classical molecular dynamics equations introduce statistical fluctuations in the data. As a result, it is difficult to determine exactly how many defects a new cascade event would produce in a complex microstructure, and even define the nature of these defects. In view of this, it might be desirable to formulate and explore a model that circumvents entirely the fluctuation aspect of defect production, and instead explores the evolution of microstructure driven solely by discrete deterministic defect formation events. In such a simulation, $\phi$ becomes a deterministic variable and acquires the meaning of the total number of defects generated by the algorithm over 
the duration of the simulation, divided by the total number of atoms in a simulation cell.

In what follows, we investigate the microstructure that forms as a result of application of a simplified model based on a creation-relaxation algorithm (CRA). The algorithm evolves the microstructure by randomly picking an atom in a simulation cell, and inserting it at a randomly chosen location in the same cell, according to a chosen probability distribution. This process may be considered as being similar to the creation of Frenkel pairs with the added simplification that it does not involve treating the high-energy part of the defect production process. In terms of its physical content, the highly simplified nature of the algorithm makes it similar to the Ising model for phase transitions or the sand-pile model for self-organized criticality [55]. Still, the algorithm fully takes into account the effects of linear and nonlinear lattice deformation resulting from the accumulation of defects, and hence enables treating the aspect of the evolution problem that has so far proved most challenging to kMC simulations. By not considering the highenergy stages of radiation damage production, the algorithm minimizes the computational effort, enabling the exploration of high values of $\phi$ even for relatively large simulation cells.

Despite the above seemingly strong model assumptions, the complexity of defect and dislocation microstructure emerging from a CRA simulation is similar to the microstructure found in full cascade simulations, with the added benefit of that a CRA analysis features a somewhat lower level of statistical noise. In addition, the CRA analysis of the highdose limit enables answering a question that had so far proved elusive, namely, what is the maximum density of defects that a material exposed to irradiation can accumulate at a relatively low temperature. CRA simulations show that, depending on the material and the adopted form of the interatomic interaction law, this asymptotic defect concentration is in the several percent range.

The CRA algorithm may be defined as follows.

(1) We construct a lattice of $N$ atoms of a desired size and crystallography.

(2) Set $N_{\mathrm{FI}}=0$, where FI refers to a Frenkel pair insertion.

(3) Randomly choose one atom.

(4) Randomly choose a position within the simulation cell according to a desired spatial probability distribution.

(5) Place the chosen atom at the new position.

(6) Use an energy minimization method to find a new (zero-force) global potential energy minimum for all the atoms in the simulation cell.

(7) Set $N_{\mathrm{FI}}=N_{\mathrm{FI}}+1$, and update the canonical value of DPA parameter $\phi$ to $N_{\mathrm{FI}} / N$.

(8) Repeat steps 3 to 6 until the algorithm reaches a desired end of simulation value of canonical DPA.

In its form given above, the CRA describes a high dissipation mode of defect production, effectively corresponding to zero temperature, and thus describes a regime for which the defect generation rate is always higher than the inverse relaxation timescale associated with the thermally activated microstructural evolution. In this limit the canonical DPA represents a natural choice of a parameter describing defect production, and in what follows will be referred to as cDPA. An algorithm similar to CRA has recently been applied to the investigation of accumulation of defects in pure iron by
Chartier and Marinica [56]. While the study was primarily focused on the exploration of the interplay between the dislocation microstructure and small inclusions of the Laves C15 phase, the authors of Ref. [56] noted several remarkable features of microstructural evolution, including a nonmonotonic variation of the density of dislocations across the DPA range from 0.01 to $1 \mathrm{cDPA}$, and the emergence of an asymptotic microstructural state that we explore here in detail. By comparing tungsten and iron, we show that the dynamic microstructural transition occurring in the range of doses close to $0.1 \mathrm{cDPA}$ is a general phenomenon, unrelated to the presence of the $\mathrm{C} 15$ phase in the material. Work has also been done on modeling HCP $\mathrm{Zr}$ at finite temperature starting with either a perfect lattice or an initial defect structure [57]. This work focused on defect cluster dynamics and revealed a correlation between defect content and volume expansion, suggesting a link to prebreakaway volume swelling. A similar approach has also been taken by Chartier et al. [58] for urania, in which they studied microstructural evolution as a steady state was approached. Algorithms involving the generation of individual defects have also been used to investigate dislocation evolution in the vicinity of $\mathrm{Zr}-\mathrm{Nb}$ multilayer phase boundaries [59], amorphization in $\mathrm{Cu}-\mathrm{Ti}$ intermetallic compounds [60], and model Lennard-Jones systems [61]. Studies addressing small doses or small system sizes can be found in Refs. [62-64].

In the absence of thermal activation, what drives the evolution of microstructure? Surprisingly, it is possible to give an unambiguous answer to this question. Indeed, what drives structural atomic relaxation in the absence of thermally activated motion is the direct nonlinear response of structure to forces acting between the atoms. The relaxation of microstructure on the scale of an entire simulation cell is driven by a spatially fluctuating stress developing in the cell as a result of spatially varying deformation (strain) stemming from the production of defects. In this respect, the evolution predicted by the CRA algorithm is fundamentally similar to the evolution observed in a dissipative discrete dislocation dynamics simulation, or in a simulation of an earthquake. An apparent similarity between earthquakes and collision cascade events was noted earlier $[18,55]$ in relation to the power law statistics found in high-energy atomic impact events [65].

The present study is organized as follows. We start by describing the computational details of the creation-relaxation algorithm. Given that the notion of canonical DPA provides a precise nonfluctuating measure of radiation exposure, we investigate the stages of evolution of microstructure from a perfect crystalline state to the yet unknown asymptotic structural form emerging in the limit cDPA $\rightarrow \infty$. Although intuitively it might appear likely that a crystalline material subjected to repeated events of production of defects should eventually reach an asymptotic dynamic equilibrium state, which is statistically invariant with respect to the continuing generation of new defects, the structure of such a state has never been determined. We find that this asymptotic dynamic equilibrium state is characterized by strong fluctuations of stress and strain, while still largely retaining crystalline order. The effective predicted defect content of the asymptotic state amounts to several percent of lattice sites. CRA simulations show that this structurally fluctuating state evolves from the 
initial pure crystalline state through several stages, spanning intervals to $\phi \sim 0.01 \mathrm{cDPA}$, then $\phi \sim 0.1 \mathrm{cDPA}$, and then $\phi \sim 0.7$ cDPA, eventually reaching an asymptotic structural configuration in the high-dose limit. The asymptotic state exhibits a number of unusual properties including a remarkable nonlocal response to perturbations, and the occurrence of earthquakelike phenomena [55] where the creation of a new defect at a certain point gives rise to a major relaxation event at a large distance from the original location. We conclude with a discussion of how the properties of the asymptotic state derived from simulations can be related to experimental observations, and in particular to the problem of the so-called invisible damage in structural nuclear materials exposed to neutron irradiation.

\section{SIMULATION METHOD}

In this study, we consider a BCC lattice and a uniform spatial probability distribution of generation of defects. Thus, at each step of execution of the CRA, a chosen atom can be displaced to anywhere within the simulation cell. Upon insertion of a Frenkel pair (steps 2 to 4 of our CRA alogrithm), the conjugate gradient minimization is employed to relax the structure and find a new local potential energy minimum. In the present work, this is performed under fixed volume conditions. The use of a fixed zero pressure boundary conditions is model dependent and problematic for energy minimization algorithms because the objective function with respect to atomic positions is modified upon a change in cell volume and cell shape. Use of fixed volume will result in an evolving global stress with respect to irradiation, whereas fixed zero pressure would result in an evolving simulation cell shape and therefore explicit volumetric swelling. Fixed volume simulations do however provide an estimate of swelling through expressing the global hydrostatic pressure in units of the empirical potential bulk modulus. It is noted that some CRA simulations were performed under fixed zero pressure conditions, and the results obtained where in complete qualitative agreement with those derived from the fixed volume simulations.

For each FI, the location of the displaced atom is entirely random. If the employed empirical potential has a strong short-range repulsive form (which is usually the case for interatomic potentials used in the modeling of radiation damage), the relaxation procedure results in a physically meaningful low energy structure. For potentials that do not have this short-range feature, the initial position of the displaced atom must avoid locations too close to other atoms. This would also be necessary when using density functional theory methods to perform the relaxation. For these latter two cases (not considered here), an additional parameter is needed to define the CRA approach, giving the minimum distance between the re-inserted atom and all other atoms.

As model materials studied using the algorithm, we consider both iron $(\mathrm{Fe})$ and tungsten $(\mathrm{W})$. To simulate the atomic structure of $\mathrm{Fe}$, the embedded atom method (EAM) potential of Mendelev et al. [66] (EAM2) and the second-generation magnetic potential of Chiesa et al. [67] are used. For W, the EAM potentials of Marinica et al. [68] (EAM4) and Mason et al. [69] are used. All the simulations are performed at fixed volume since this defines a physically unambiguous

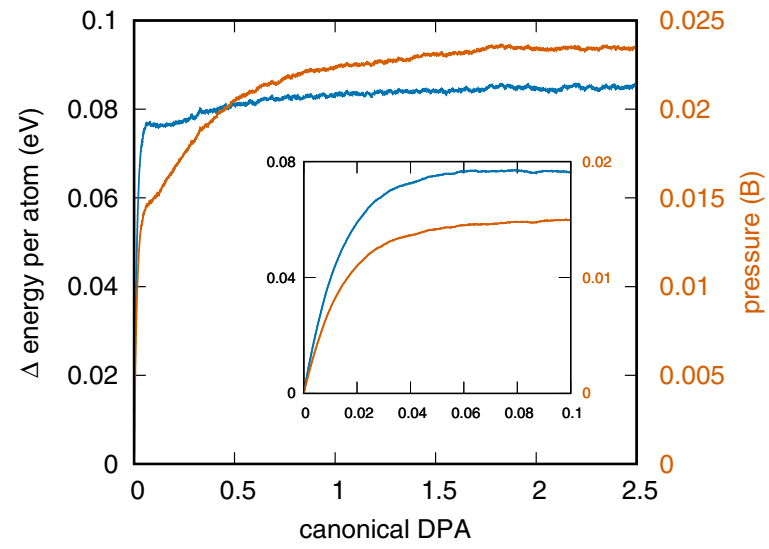

FIG. 2. Plot of the average change in energy per atom (left axis) and global pressure (right axis) as a function of canonical DPA (Frenkel pair insertion number divided by the total number of atoms). The pressure is plotted in units of the perfect BCC crystal bulk modulus of the empirical potential. The inset highlights early stages of irradiation, corresponding to low cDPA $\phi \ll 1$.

Hamiltonian equations of atomic motion. All the energy scales involved match the energy scales at which the interatomic potentials were fitted, minimizing the uncertainties associated with the simulations. The initial BCC lattice sizes considered here span cubic conventional cells with side lengths equal to $10,20,30,40$, and $80 \mathrm{BCC}$ unit cells. The largest system size considered therefore contains 1024000 atoms. In Secs. III and VI, the work focuses on the largest system size considered using the Mendelev et al. [66] potential for Fe, whereas Appendix A investigates the effect of simulation cell size for this Fe potential, Appendix B the dependence on the choice of Fe potential, and Appendix $\mathrm{C}$ considers CRA simulations of $\mathrm{W}$. The present work employs LAMMPS [70] to perform the structural relaxation and OVITO [71] for the atomic visualization and structural analysis.

\section{STRUCTURAL EVOLUTION UP TO 2.5 CDPA}

The CRA approach is now applied to explore irradiation doses up to $2.5 \mathrm{cDPA}$ in a model BCC Fe material described by the empirical intertomic potential of Mendelev et al. [66]. Results are presented for a relatively large system size. This sample contains 1024000 atoms and the simulation therefore involves 2560000 Frenkel pair insertions to reach the dose of $\phi=2.5$ cDPA. Figure 2 displays the evolution of the change in energy per atom and the global pressure as a function of cDPA. The data reveal that after an initial rapid rise, both quantities change little beyond a cDPA of $\phi \sim 1.5$, indicating that a steady state has being reached. The hydrostatic pressure is given in units of the empirical potential's bulk modulus, $G=179 \mathrm{GPa}$, giving an estimate of the equivalent volume expansion that would occur under zero-pressure irradiation conditions. Thus a cDPA value beyond unity results in a steady-state swelling of $\sim 2 \%-2.5 \%$ for our model BCC Fe. This simple estimate is within $10 \%$ of the volume swelling of the final atomic configuration at $\phi=2.5 \mathrm{cDPA}$, obtained using a combined structural and simulation cell relaxation at fixed zero pressure-see Sec. VI. 
The global shear stress components of the fixed cubic simulation cell (these components are not shown in Fig. 2) fluctuate around zero with respect to cDPA at a stress scale $\pm 5 \%$ of the hydrostatic pressure. From a systematic investigation of the steady state as a function of system size, the off-diagonal shear components of the global stress tensor scale as functions of the volume of the cell $V$ as $\sigma_{x y} \sim V^{-2 / 3}$, and are thus expected to vanish in the macroscopic limit.

Due to repeated Frenkel pair insertions, the vacancy and interstitial content gradually increases. To detect the interstitial and vacancy content, the Wigner-Seitz method is used. Here, an array of Wigner-Seitz (Voronoi) volumes, corresponding to the initial BCC lattice, is constructed as a reference. To determine the interstitial/vacancy defect content of an atomic configuration at a particular dose, the number of atoms within each Wigner-Seitz volume is counted. A Wigner-Seitz volume containing no atom is identified as a vacancy, and when it contains two atoms, it is identified as a self-interstitial. A higher number of particles could be contained within a Wigner-Seitz volume, however the present work found this to be extremely rare. Because of particle conservation, the instantaneous total numbers of vacancies and self-interstitials, in what follows referred to as interstitials, measured this way are always found to be equal. However, such a method of counting defects does not distinguish between isolated interstitial defects and, for example, an extra full plane of atoms associated with a planar disk of interstitials (an interstitial dislocation loop). Inspection of the bond distance for the identified interstitials allows this distinction to be approximately made. If the bond distance is comparable to the nearest-neighbor distance in the perfect lattice, the interstitials are identified as belonging to a large dislocation loop or an extra plane of atoms. If the bond distance is significantly less than the value expected for the perfect lattice, $\sqrt{3} l_{0} / 2$, where $l_{0}$ is the BCC lattice parameter, then the interstitial is identified as an isolated defect. The upper range is taken as 0.93 that of $\sqrt{3} l_{0} / 2$.

Visual inspection (not shown) of the resulting lattice interstitials reveal them to be well-separated from those interstitials contributing to the extra atomic planes associated with dislocation loops. The approach taken can not resolve other possible structures such as mixed Laves C15/dislocation loop structures-an aspect not considered in the present work. Indeed, a common-neighbor analysis of the evolving microstructure revealed only a very small icoshadral content during the dislocation loop nucleation regime, where in a few cases small clusters of Laves back-bone polyhedral structures were observed. The much larger clusters seen in the work of Chartier and Marinica [56] were not found.

Figure 3 displays the evolution of the interstitial/vacancy percentage content as a function of cDPA. At lower cDPA values, the interstitial and vacancy content is seen to be identical and to rise rapidly. At a cDPA value close to $\phi \sim$ 0.05 , the isolated interstitital defect content diverges from that of the vacancy and begins to decrease. At the highest cDPA values, the vacancy content plateaus at approximately 3.5\% and the isolated interstitial content at approximately $0.5 \%$, highlighting the unusual nature of the microstructure of the steady state identified via energy and pressure. A qualitatively similar trend was observed in simulations of radiation damage effects in urania [58].

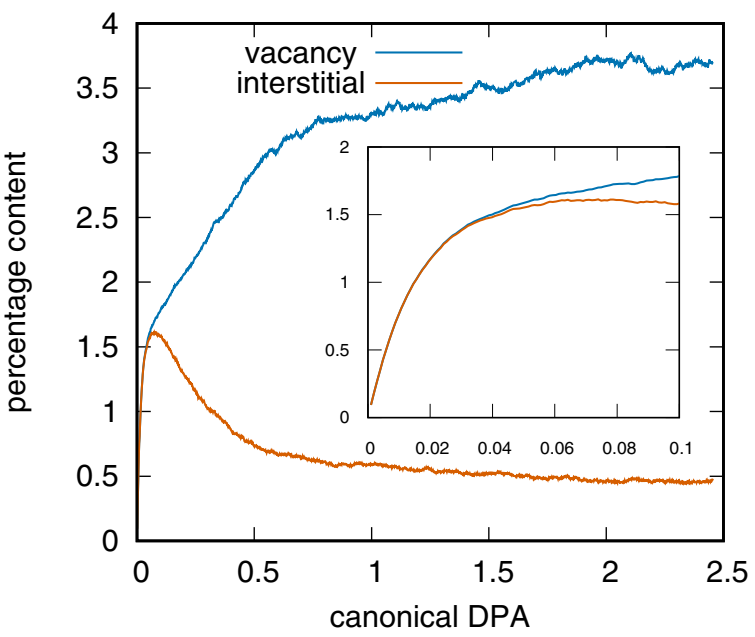

FIG. 3. Plot of vacancy and individual interstitial percentage content as a function of canonical DPA (Frenkel pair insertion number divided by the total number of atoms). The inset highlights the early stages of irradiation corresponding to low cDPA.

Together, Figs. 2 and 3 show that at a sufficiently high-dose corresponding to values above $\sim 2 \mathrm{cDPA}$, an approximate steady state is reached in terms of energy per atom, global hydrostatic pressure, and interstitial/vacancy defect content. Under zero-pressure conditions this would entail a steadystate swelling of $\sim 2 \%$. This latter aspect will be considered in more detail in Sec. VI.

In the context of the apparent emergence of a microstructurally complex steady state in the high cDPA limit, it would be appropriate to note a kMC study of the accumulation of damage by Björkas et al. [72] who observed that, due to recombination, the density of radiation defects saturates at a relatively low dose $\phi \sim 0.01$ if a $\mathrm{kMC}$ model takes into account the trapping of defects by impurities. While the kMC study [72] did not take into account the formation of an extended dislocation network, which as we show below is highly significant, the effect of trapping of defects by impurities is pertinent and consistent with the fundamentals of the study presented here. Interaction between defects and impurities immobilize radiation defects $[50,73,74]$, and this extends the range of validity of the model considered here further into the finite temperature domain.

The insets in Figs. 2 and 3, which focus on doses of up to $0.1 \mathrm{cDPA}$, exhibit an initial linear dependence on cDPA for all quantities. This underlies the additive nature of the FI effect in the dilute defect limit, where each new vacancy and interstitial is created in a nominally perfect BCC lattice environment. Such a linear dependence and the associated dilute regime do not last, and a significant deviation from linearity is already observed at cDPA values close to $\phi \sim 0.02$. In this transition regime, the energy per atom slightly decreases over a short cDPA interval during which the isolated interstitial content begins to deviate from the vacancy content. Thus the BCC lattice appears to momentarily regenerate. A detailed inspection of the corresponding atomic configurations as a function of cDPA reveals that this apparent regeneration is due to the onset of clustering of interstitials into dislocation loops. This 


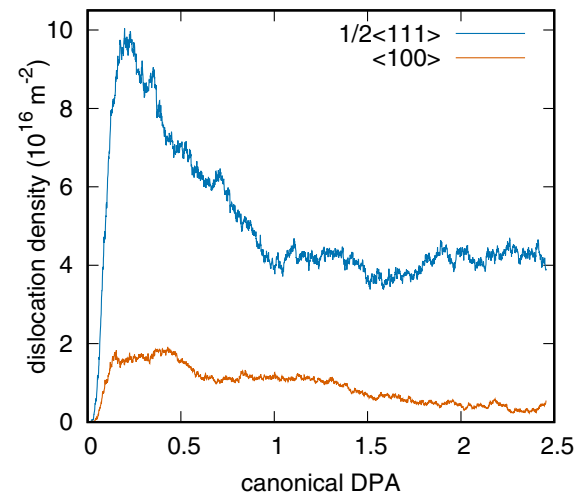

FIG. 4. Plot of the evolving density of dislocations with Burgers vectors $1 / 2\langle 111\rangle$ and $\langle 100\rangle$ shown as a function of canonical DPA (Frenkel pair insertion number divided by the total number of atoms).

process in turn explains why the isolated interstitial content is now less than that of the vacancies and why it is seemingly decreasing. Thus there exists a transition from isolated interstitials to interstitial dislocation loops, which upon further FIs grow in size. This may be seen as a new dilute regime, now of relatively isolated interstitial loops, corresponding to a fundamentally new transient stage, also characterized by (approximately) linear behavior of energy per atom, global hydrostatic pressure, and point defect content with respect to increasing cDPA. This second regime of structural response to exposure to irradiation continues up to $\phi \sim 0.5 \mathrm{cDPA}$.

The aforementioned transition and the final approach to a steady-state defect structure, with respect to increasing irradiation dose, is best seen through the corresponding dislocation evolution. Figure 4 shows the dislocation density as a function of cDPA. Here, OVITO is used to identify the dislocation content according to the algorithm outlined in Refs. [75,76]. Only negligible $\langle 110\rangle$ dislocation content was observed. For the Mendelev potential, $1 / 2\langle 111\rangle$ dislocation loops are lower in energy in comparison to $\langle 100\rangle$ loops of similar size, suggesting a simple reason why $1 / 2\langle 111\rangle$ loops dominate over $\langle 100\rangle$ loops.

The data show that dislocation content emerges as a new order parameter characterising the microstructure at approximately $0.02 \mathrm{cDPA}$, and is associated with the formation of small $1 / 2\langle 111\rangle$ and $\langle 100\rangle$ interstitial dislocation loops. Figure 4 also indicates a maximum in the dislocation density at a cDPA of $0.2-0.3$, corresponding to a deviation away from the second linear defect evolution stage and marking the end of the dilute dislocation loop growth regime. At a cDPA value of about 0.5, a third and final approximate linear regime is entered, which may be associated with the coalescence of interstitial dislocation loops, and the beginning of the development of an extended system-spanning dislocation network. The presence of a maximum of dislocation density at a dose close to $\phi \sim 0.1 \mathrm{cDPA}$ was also noted in a study by Chartier and Marinica [56].

Thus the CRA model, through multiple FIs at $0 \mathrm{~K}$, is able to produce a physically meaningful structural evolution trajectory from an initially perfect crystal lattice at $\phi=0$ to a steady state characterized by the co-existence of isolated defects, defect clusters, and extended dislocation network at irradiation doses $\phi \gg 1$ over an interval of cDPA values of $O(1)$. A similar structural evolution towards high doses was identified for urania [58].

Figure 5 displays atomic-scale visualizations of the evolving microstructure. In these pictures, the left panels (blue balls) represent vacancy sites, the central panels (red balls) represent interstitial defect sites, and the right panels the $1 / 2\langle 111\rangle$ (green lines) and $\langle 100\rangle$ (purple lines) dislocation structures. Figure 5(a) shows the configuration at $0.02 \mathrm{cDPA}$ corresponding to the very onset of dislocation loop formation. At this dose, only one dislocation loop (see arrow in rightmost panel), with the Burgers vector of $1 / 2\langle 111\rangle$, is seen and the vacancy and isolated interstitial contents are still identical. Figure 5(b) shows a configuration at $\phi=0.1 \mathrm{cDPA}$, corresponding to the dilute dislocation loop regime, and the dislocation density that is about half that of the maximum achieved later at a cDPA value of 0.2. An approximately uniform density of loops is seen with Burgers vectors $1 / 2\langle 111\rangle$ and $\langle 100\rangle$. At this cDPA value, the isolated interstitial and vacancy defect contents begin to diverge from each other, with the vacancy content increasing and the isolated interstitial content decreasing. Figure 5(c) now shows configurations at $0.2 \mathrm{cDPA}$, corresponding to the peak in the dislocation line density seen in Fig. 4. The microstructure now contains larger dislocation loops that originate from the coalescence of smaller loops. Some dislocation structures are seen that may no longer be easily identified as dislocation loops, indicating the start of the development of an extended dislocation network. Here also, the isolated interstitial content is clearly lower than that of the vacancy. Figure 5(d) shows the final asymptotic configuration at $2.5 \mathrm{cDPA}$, which is well within the steady-state regime. Here, system spanning $1 / 2\langle 111\rangle$ dislocation structures are seen along with a significantly reduced population of dislocation loops, all of which are embedded in a spatially uniform dense population of vacancies and isolated interstitials.

The left panels of Fig. 5 demonstrates that up to a value of $2.5 \mathrm{cDPA}$, the spatial distribution of vacancies remains considerably homogeneous, a result that is expected given the lack of thermally assisted vacancy diffusion. Such a homogeneity is also seen in the spatial distribution of lattice interstitials (central panels of Fig. 5), that is, those interstitials that do not become part of the extra atomic planes associated with the dislocation network.

The observation of a significantly reduced isolated interstitial population indicate that a considerable fraction of self-interstitials produced through FIs exist now as the extra lattice planes associated with the observed dislocation content. The vacancy content of the steady-state microstructure is therefore unusually high, a prediction that appears to be in good agreement with experimental observations [77]. A natural conclusion of the fact that the density of defects is high is that the emerging steady-state microstructures are characterized by a significant internal strain, and therefore internal stress. These are material state variables that will be shown to play a central role in the characterization of the steady state. The occurrence of large internal stress and strain in a material containing dense populations of radiation defects agrees with the elasticity analysis performed in Ref. [78]. 
(a)

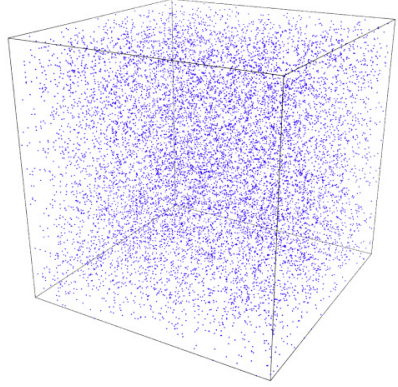

(b)

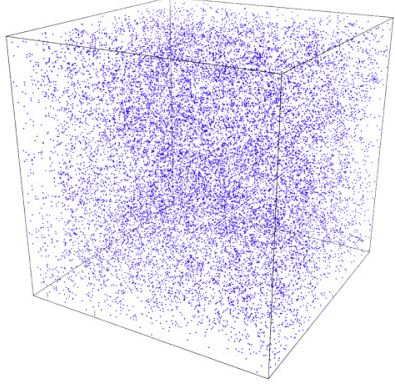

(c)

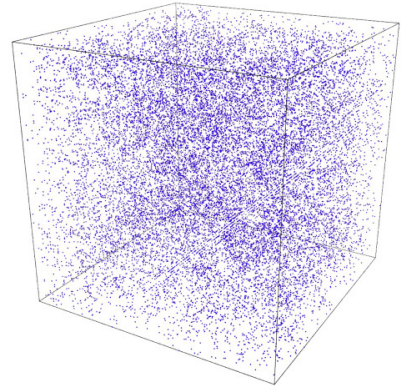

(d)

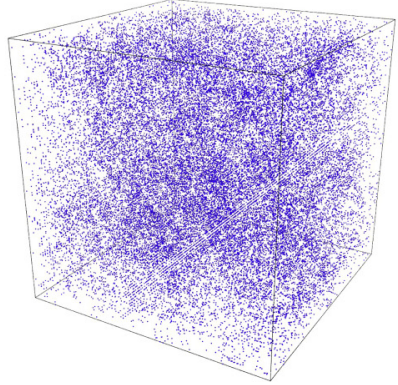

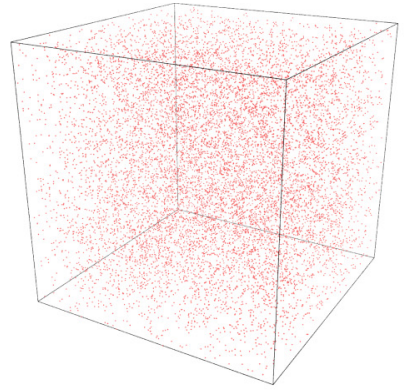
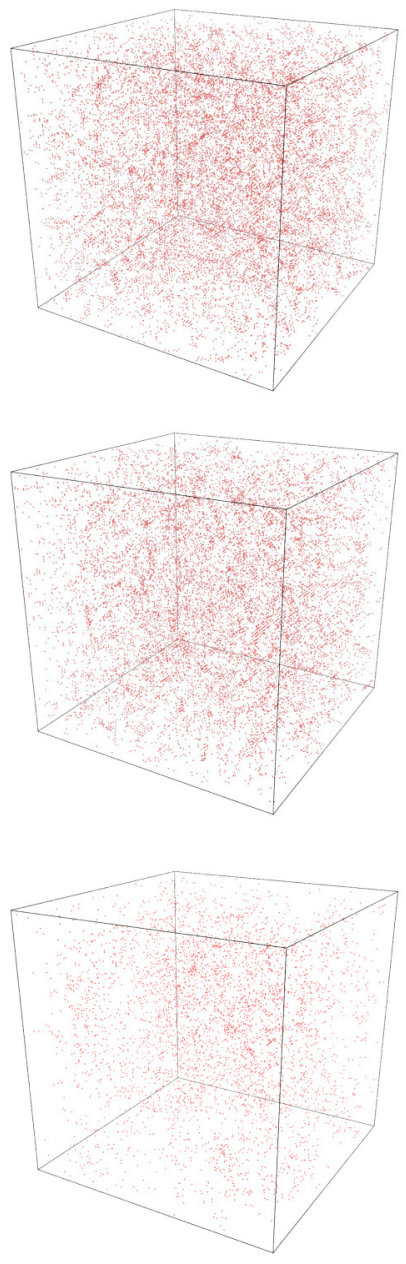
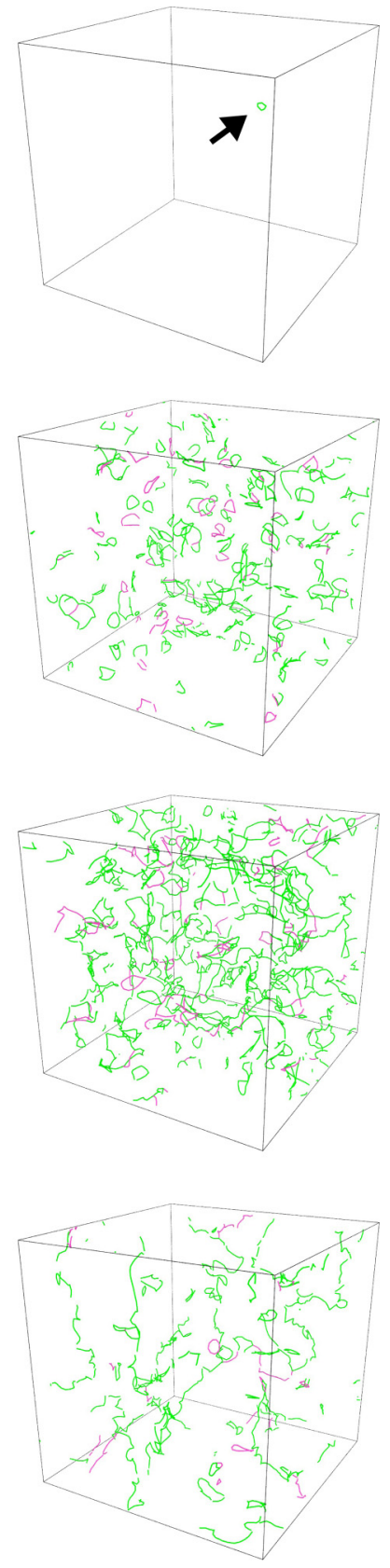

FIG. 5. Atomistic and dislocation visualization of BCC Fe microstructural configurations at cDPA values of (a) 0.02: the linear FI regime, (b) 0.1 : the onset of dislocation loop nucleation and growth, (c) 0.2 : dislocation coalescence and the onset of formation of an extended dislocation network, and (d) 2.5: the steady state of an extended (system-spanning) dislocation network. The left panels display the vacancy content, the central panels the isolated interstitial content, and the right panels the dislocation content. Note the particularly high vacancy content of the microstructure corresponding to $2.5 \mathrm{cDPA}$, where the isolated interstitial and dislocation density content are both relatively low. This vacancy content is balanced by self-interstitials contained in the system-spanning dislocation network. The steady-state configuration shown in (d) has the highest volume (upon cell relaxation at fixed zero stress, see Sec. VI), owing to defect-induced swelling.

\section{THE STEADY STATE}

The results of Sec. III indicate that the initially perfect crystal gradually evolves into an microstructural state that appears effectively resistant to irradiation and statistically invariant with respect to multiple FIs. This microstructural state emerges as a result of application of the CRA algorithm introduced in Sec. I. In terms of global variables displayed in Figs. 2-6, this steady state may be characterized by the spatially fluctuating energy density and global stress that are intimately related to the corresponding fluctuations in the microstructure, defined by its variable and spatially varying interstitial, vacancy and dislocation content.

In the steady state, exposure-independent microstructure, it becomes statistically meaningful to define a probability distribution of material response. We start by exploring the distribution of events associated with changes in the total energy upon a FI insertion. Figure 6(a) plots an un-normalized 


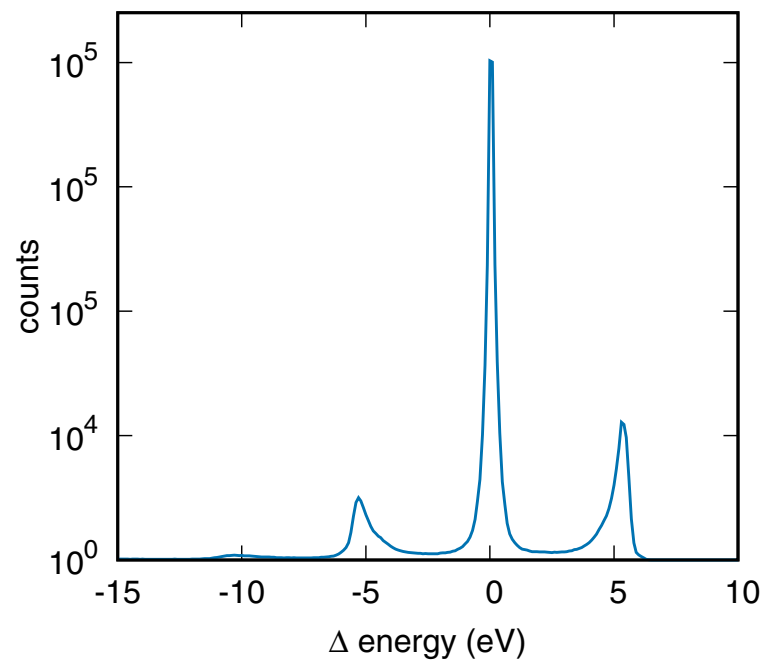

(a)

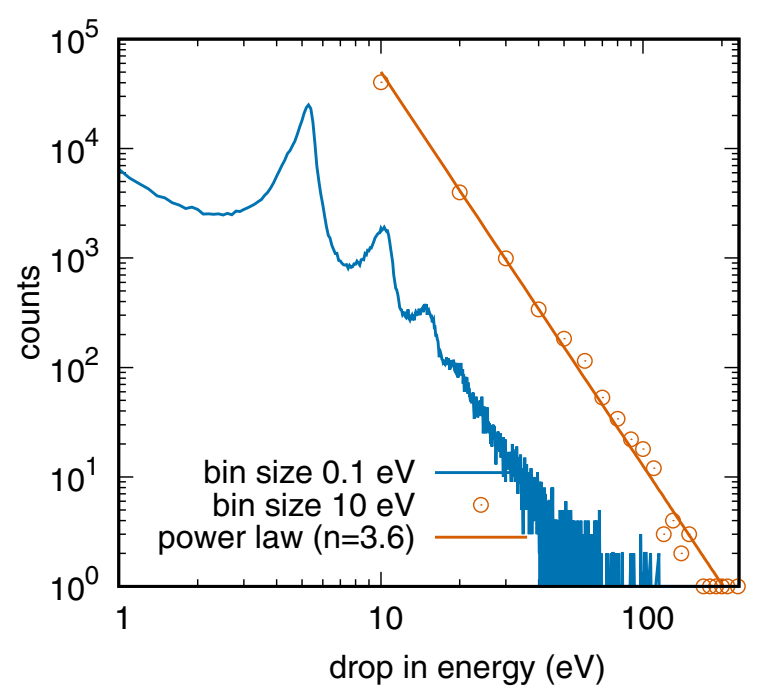

(b)

FIG. 6. (a) Histogram derived from the statistical analysis of energy changes due to a single Frenkel insertion performed in the steady-state regime. (b) Same data for the negative domain (energy drop) as a log-log plot indicating a periodically peaked distribution corresponding to multiple Frenkel pair annihilation that merges to a power-law tail with an exponent equal to approximately 3.6.

histogram of total energy changes resulting from individual FIs in the steady state. The data are derived from approximately 1000000 FIs at cDPA values higher than 1.5. The first moment of the histogram is close to zero, ensuring that the stationary state with respect to total energy is maintained. The figure reveals a central peak at zero energy, and two smaller peaks at energies of $\pm 5 \mathrm{eV}$. There is also a third peak at $10 \mathrm{eV}$ that is better seen in a log-log plot of the negative domain of the distribution [Fig. 6(b)]. Inspection of Fig. 6(b) reveals two further peaks of decreasing intensity, again located at multiples of $5 \mathrm{eV}$. Such peaks are due either to the creation or annihilation of (multiple) Frenkel pairs, where for the Mendelev potential the energy of an isolated Frenkel pair in the otherwise perfect BCC lattice is $5.24 \mathrm{eV}$.

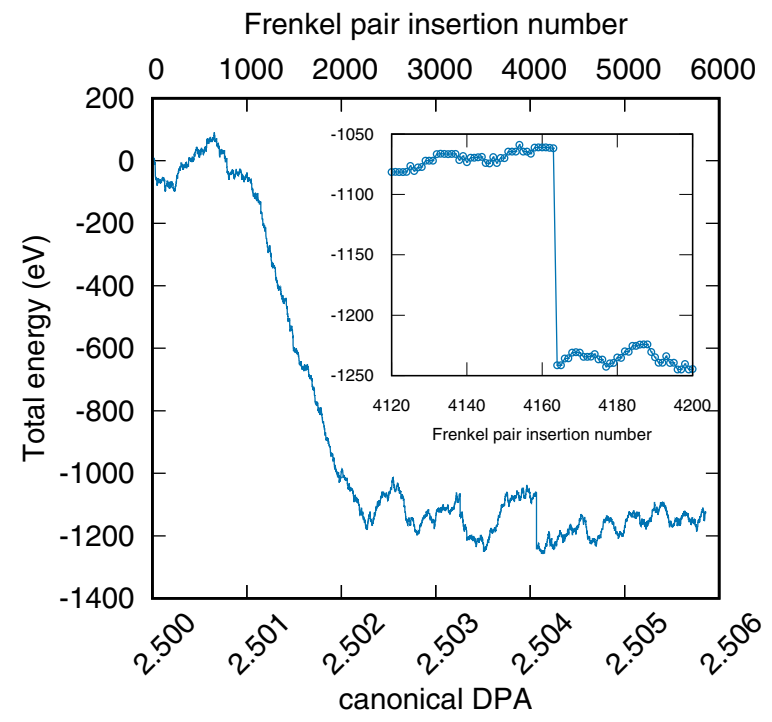

FIG. 7. Snapshot of the total energy plotted as a function of canonical DPA (Frenkel pair insertion number divided by the total number of atoms) within the steady-state regime. The upper horizontal axis shows the total energy as a function of the actual Frenkel pair insertion number. Both energy and Frenkel pair insertion number are relative to the starting configuration taken at a cDPA of 2.5. The inset shows a zoom-in, as just a function of the Frenkel pair insertion number, of a region of the curve that exhibits a larger energy drop of $200 \mathrm{eV}$ upon a single Frenkel pair insertion.

Figure 6 indicates that the most probable response of the material to a FI is a negligible change in energy. This originates either from the randomly displaced atom filling an existing vacancy site, or from the annihilation of an existing Frenkel pair. The next likely responses are either a rise in energy due to the creation of an additional vacancy and interstitial directly from the FI, or a drop in energy corresponding to multiple Frenkel pair annihilation. It is the balance of these three possible responses to a new FI that ensures the steady-state nature of the microstructure developing in the $\phi \gg 1$ limit.

The response mode involving multiple Frenkel pair annihilations is quite remarkable, for it suggests a collective and therefore less local stress relaxation event involving many atoms. Figure 6(b) shows that such a response populates the power-law tail of the histogram and can entail an energy drop of up to $O(100 \mathrm{eV})$-an asymptotic regime in which the peak-structure associated with Frenkel pair annihilation is completely lost within the log-log scale of the current statistical data. The observed power law of $\sim 1 / \Delta E^{3.6}$ indicates that such collective response events are relatively rare, but not exponentially rare as would have been the case for a Gaussian distribution of energy drops. This non-Gaussian feature of the histogram reflects a nontrivial potential energy landscape characterized by energy and length scales well beyond those associated with an isolated microscopic Frenkel pair defect.

Figure 7 displays the total energy change as a function of the FI number, beginning at a cDPA value of 2.5. Two scales of change-in-energy are seen. The first involves changes in energy associated with each FI, the statistics of which are described by the histograms of Fig. 6. The inset of Fig. 7 


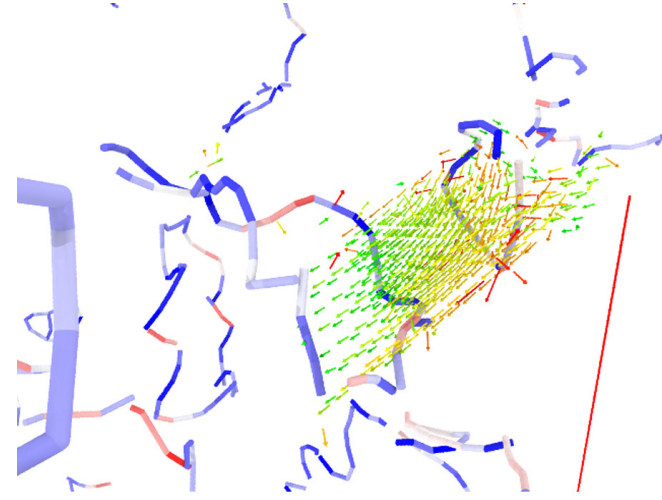

(a)

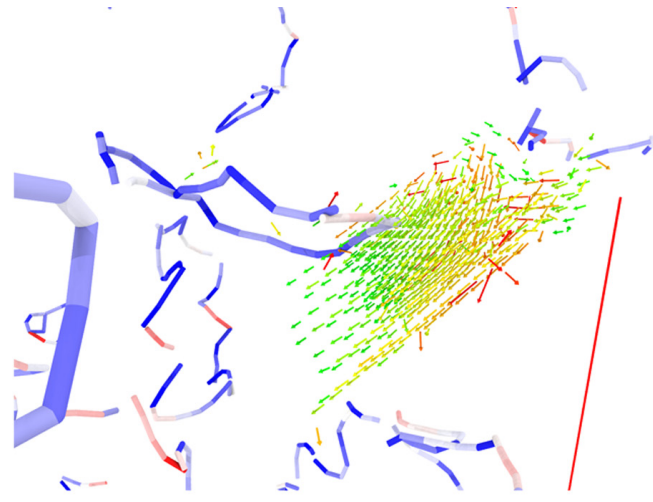

(b)

FIG. 8. Visualization of a region in which only the dislocation lines are shown, which are identified according to the algorithm of Stukowski et al. $[75,76]$. The shade of blue indicates the character of the identified segments where blue represents edge character and red represents screw character. (a) Dislocation structure prior to Frenkel pair insertion number 4163 (see Fig. 7) and (b) dislocation structure after the insertion. Both panels include atomic displacements greater than $0.1 \AA$ between these two configurations, with the color corresponding to the atoms displacement magnitude. The long red vector to the right of each panel indicates the displacement associated with the Frenkel pair insertion. Only part of the of the vector is seen, with the upper end showing the location of the created vacancy defect.

displays a zoomed-in region where a single FI has caused a drop in energy of almost $200 \mathrm{eV}$. Detailed inspection of the corresponding atomic arrangements reveals a structural response occurring over a spatial volume of several nanometers, in a region far from the final position of the displaced atom and about a nanometer from the vacancy created by the FI. This is visualized in Figs. 8(a) and 8(b), which show the dislocation content (a) before and (b) after the FI. Also shown in both (a) and (b) are the atomic displacements greater than $0.1 \AA$, which occur between the two atomic configurations. The figure demonstrates that the drop in energy of almost $200 \mathrm{eV}$ originates from a major stress-driven reorganisation of the dislocation structure, in which a dislocation loop moves and coalesces with a larger nearby loop.

Figure 9 now displays the entire simulation cell in which only those atoms that undergo a displacement greater than $0.01 \AA$ are shown. Each atom is colored according to its displacement, with blue representing $O(0.01 \AA)$ displacements and red $O(1.0 \AA)$ displacements. These larger displacements are centered on the structural transformation process described in Fig. 8. The figure shows that the spatial extent of the displacement field has a dominant quadrupole symmetry-a result that is theoretically expected from the far-field elastic stress and strain field of a localized defect structure, see, e.g., equations (18) and (22) of Ref. [78].

The second fluctuation energy scale seen in Fig. 7 is associated with a $O(1000 \mathrm{eV})$ energy drop equivalent to the annihilation of approximately one thousand FIs, note the large drop in energy occurring between FI numbers 1000 to 2000. This extended regime of microstructural evolution is dominated by a large number of sequential relatively small energy drops, each corresponding to the annihilation of one or two Frenkel pairs per FI. A similar figure to Fig. 9 is shown in Fig. 10, where now the two configurations are at FI numbers 1300 and 1400. An inspection of Fig. 10 reveals a spatially homogeneous distribution of local displacement fields, where each has a pronounced quadrupolar symmetry.
The observation that a sequence of several hundred Frenkel pair insertions produces an energy reduction suggests that the microstructure is characterized by a potential energy landscape whose valleys and ridges are separated by energies on the $O(1000 \mathrm{eV})$ scale. Such a large energy variation is "rippled" by the local energy minima of the stable configurations between each FI, and thus involving an energy scale of $O(1 \mathrm{eV})$. It is noted that a similar behavior is seen for comparable increases in energy, which is an expected result under the statistically steady-state condition. Visual inspection of

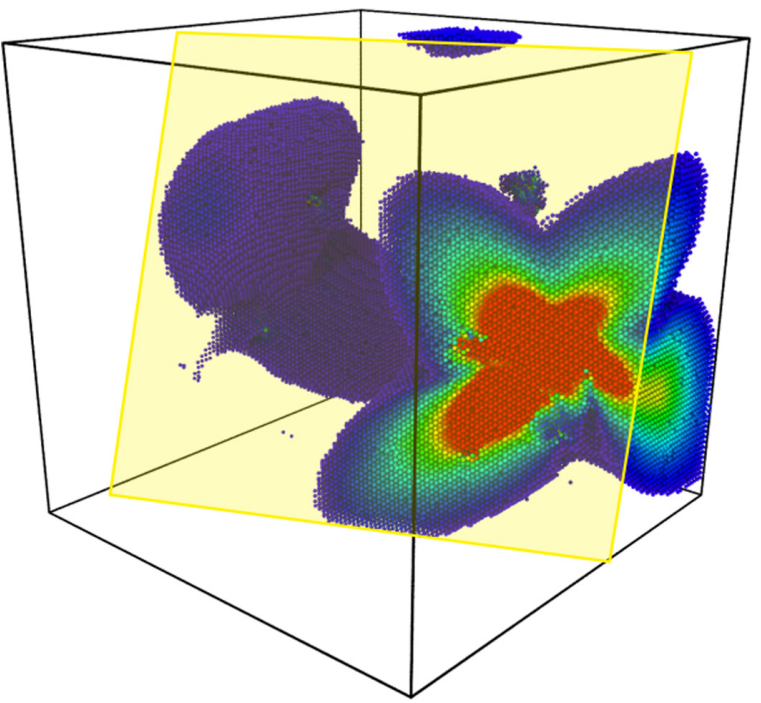

FIG. 9. Partial atomic configuration at Frenkel pair insertion number 4163 (see Fig. 7), showing only those atoms whose displacements are larger than $0.01 \AA$ when compared to the configuration at Frenkel pair insertion number 4164 . The center of the dominant quadrupolar structure is located in the region where a dislocation loop has coalesced with a neighboring larger dislocation loop. The yellow shaded plane indicates the cut plane beyond which atoms are not shown. 


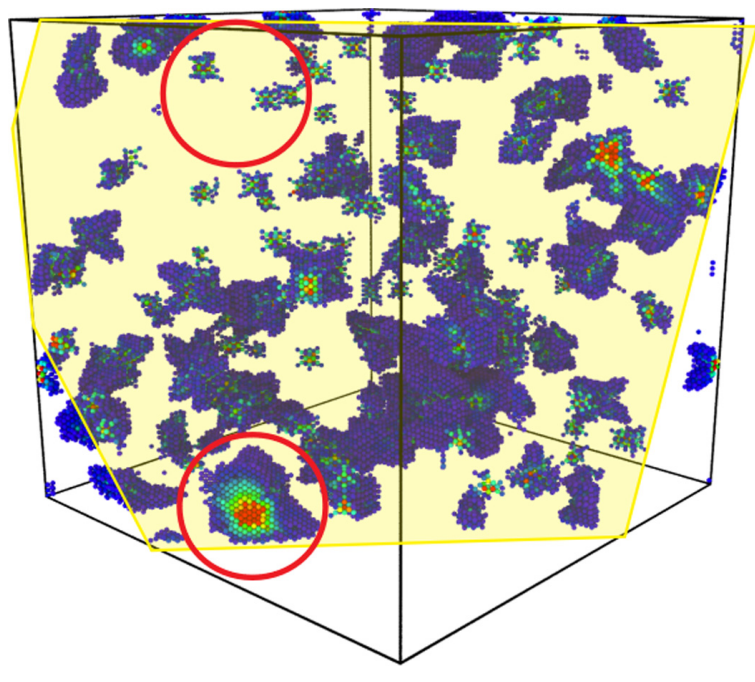

FIG. 10. Partial atomic configuration at Frenkel pair insertion number 1300 (see Fig. 7), showing only those atoms whose displacements are larger than $0.01 \AA$ when compared to the configuration at Frenkel pair insertion number 1400. The circled regions display typical local displacement fields resulting from individual Frenkel pair insertion, which can vary in size and orientation, but with a similar quadrupolar symmetry. The yellow shaded plane indicates the cut plane beyond which atoms are not shown.

the dislocation network over the FI number range from 1000 to 2000 reveals negligible change in its structure, supporting the notion that it is the dislocation network configuration that determines this larger scale of the potential energy landscape.

The changing displacement fields seen in Figs. 9 and 10 naturally entail a fluctuating internal strain and therefore a fluctuating internal stress produced by the FIs. The use of an empirical central-force model, such as the Mendelev EAM potential for $\mathrm{Fe}$, allows for a straightforward estimate of the local stress variations at the resolution of an atom [79]. Such a calculation requires a spatial definition of the local atomic volume, a quantity that is somewhat arbitrary on the scale of a bond length. However, the atomic volume enters the stress calculation as a prefactor and its calculation may be avoided by considering the logarithm of the atomic stress. Since the local stress can vary greatly over the atomic scale, the now additive log-volume term may be neglected since it varies little in comparison to the logarithmic variations of the actual stress. In what follows we follow this approach, however still refer to the displayed quantity as the logarithm of the local stress.

Figure 11 plots the calculated local stress, shown as the corresponding von Mises stress for the configurations considered so far. The von Mises stress is a scalar parameter proportional to the square root of the positive definite $J_{2}$ invariant of the $3 \times 3$ stress tensor $\hat{\sigma}, \sigma_{\mathrm{vM}}=\sqrt{3 J_{2}}$, where

$$
J_{2}=\frac{1}{2}\left[\operatorname{Tr}\left(\hat{\sigma}^{2}\right)-\frac{1}{3}(\operatorname{Tr} \hat{\sigma})^{2}\right],
$$

and represents a convenient measure of the magnitude of the stress corresponding to the largest shear strain energy [80]. In these figures, a cross-section of atoms is displayed which are colored according to the logarithm of their von Mises stress. General inspection revels a strong variation of the internal strain consisting of both large discrete values localized at dislocation cores, as well as a somewhat smoother variation occurring at the nanometer length scale. This latter component is due to the strain fields associated with lattice defects. Similar variations can be seen when the local hydrostatic pressure is considered.

Figures 11(a) and 11(b) correspond to the atomic configurations immediately before and after the FI, which causes a drop of almost $200 \mathrm{eV}$ and the associated coalescence of two dislocation loops (see Figs. 8 and 9). The chosen atomic slice intersects the spatial region where this occurs and is identified by the yellow circles. In this circled region, the local shear stress clearly changes at a length scale of several nanometers due to the changes in the elastic fields due to the dislocation reaction. Figures 11(c) and 11(d) correspond to the atomic configurations at FI number 1300 and 1400 (see Fig. 10). For this regime of behavior there is little change at the nanometer scale of the shear stress due to little change in the dislocation network and its corresponding elastic fields. However, changes are seen at the local atomic scale of the interstitials annihilated by the FIs, reflecting the predominantly local atomic structural response to FI.

The picture that therefore emerges is a steady-state microstructure whose internal stress and strain are largely determined by the fluctuating dislocation network that consists of both dislocation loops and extended dislocation line defects, as well as a population of isolated interstitial defects. This defect structure is embedded in a crystal lattice with a very high vacancy content of approximately $4 \%$. The larger energy scale of the corresponding potential energy landscape is determined primarily by the dislocation network, and may be associated with a spatial length-scale set by its line density. Superimposed on this larger-scale potential energy landscape of valleys and ridges is a smaller energy and spatial length scale of fluctuating energy minima set by the more local structures of the isolated or clustered interstitial and vacancy defects. This lower energy scale is linked to the larger energy scales by the collective nature of the dislocation network, allowing a change in the local atomic structure to induce a change at much larger energy and length-scales, as evidenced by the single FI inducing a dislocation reaction resulting in loop coalescence that we discussed above.

The persistence of the steady microstructural state with respect to further FI has been demonstrated up to a value of 20 cDPA for the smaller simulation cell sizes. Figures 12(a) and 12(b) display the pressure and isolated defect content evolution for the $30 \times 30 \times 30 \mathrm{BCC}$ unit cell system size. This size is sufficiently large for an extended network of dislocations to emerge and to remain. The figures reveal a steady state in both pressure and defect content up to $20 \mathrm{cDPA}$. Comparison of these figures to the data for the $80 \times 80 \times 80$ BCC unit cell system size (Figs. 2 and 3) show quantities that vary more when compared to the corresponding averages - see Appendix A. This is a global cell size effect that becomes increasingly relevant when the simulation cell size becomes comparable to the characteristic length scale associated with loop coalescence and dislocation network reorganisation. Indeed, for smaller system sizes, the dislocation line density can fluctuate between zero and a value comparable to that seen in Fig. 4. This is not unexpected and simply signifies the fact 

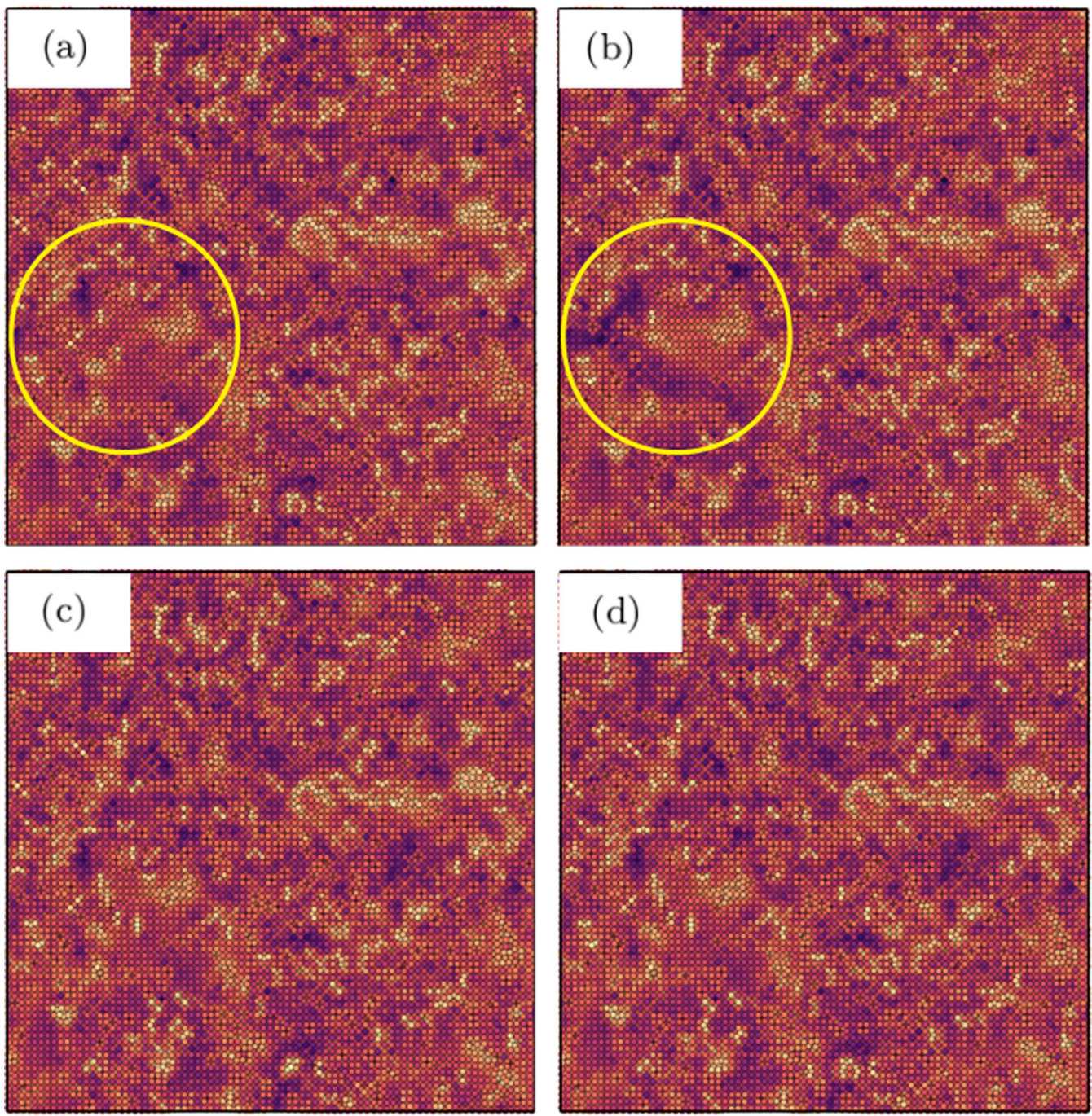

FIG. 11. Plot of atomic configurations where atoms are colored according to the logarithm of their local von Mises stress in which the darker colors refer to lower values of the logarithm. Each panel visualizes the same plane of atoms intersecting the region (circled in yellow) containing the dislocation loop coalescence event seen in Figs. 8 and 9. (a) and (b) are the configurations before and after the Frenkel pair insertion triggering the coalescence (Frenkel pair insertion numbers 4163 and 4164-see Fig. 7). (c) and (d) are for prior configurations (Frenkel pair insertion numbers 1300 and 1400_-see Fig. 7), and show the variation in shear stress due to 100 Frenkel pair insertions that do not trigger significant dislocation reorganization.

that forming full extra atomic planes in the simulation cell as a result of growth and coalescence of interstitial dislocation loops becomes easier in the relatively small cell size limit.

\section{COMPARISON TO CASCADE OVERLAP SIMULATIONS}

The multiple overlap cascade simulations by Granberg, Nordlund et al. Refs. [24,81], carried out using the Fe potential by Mendelev, provide defect evolution data up to 0.16 NRT-DPA. Figure 13 plots the interstitial percentage versus cDPA arising from the early stages of Frenkel pair insertions along with scaled data originating from the cascade overlap simulations using the same empirical potential. The original data are taken from Fig. 9 of Ref. [81], and represents the interstitial defect percentage versus NRT-DPA. The scaling used is

$$
f(x) \rightarrow a f(b x),
$$

with $a=7.0$ and $b=3.1$, resulting in a remarkable qualitative agreement between our Frenkel pair insertion and overlap cascades simulations at $300 \mathrm{~K}$. Via $b$, we now have a direct relationship between the model based NRT-DPA measure and our cDPA measure. That $a$ is not equal to unity, indicates a quantitative difference between the $0 \mathrm{~K}$ and $300 \mathrm{~K}$ conditions on the timescale of the latter, finite temperature, MD simulations. There is however no qualitative difference between the two temperatures for a regime of the early linear stage of evolution associated with the creation of initial isolated Frenkel pairs and then the nucleation and growth of small dislocation loops. This underlies the important result that despite the increased mobility of the interstitial defects at $300 \mathrm{~K}$, the basic processes seen here at $0 \mathrm{~K}$ remain the same, and also involve the creation of Frenkel pairs, the clustering of interstitials to nucleate dislocation loops, and the subsequent growth of loops leading to the formation of a dislocation 


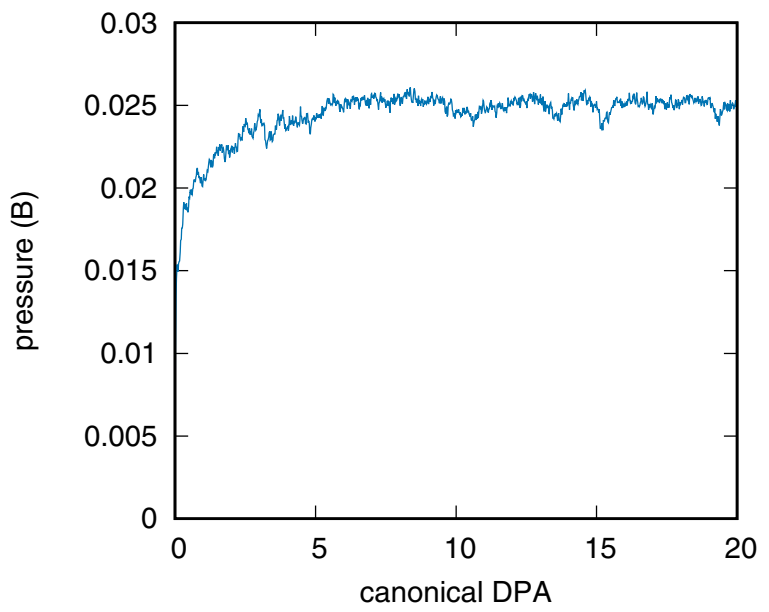

(a)

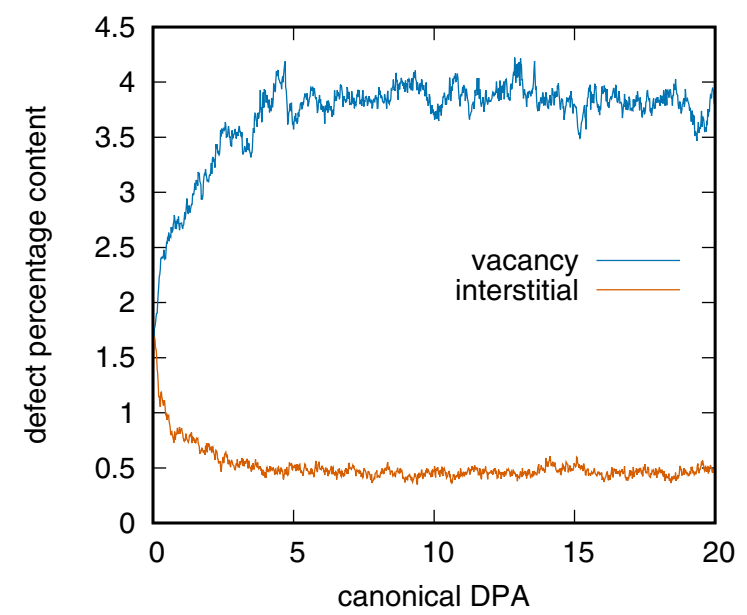

(b)

FIG. 12. Plot of (a) the global pressure and (b) isolated defect content as a function of the Frenkel pair insertion number, measured as a function of canonical DPA (Frenkel pair insertion number divided by the total number of atoms). The pressure is plotted in units of the perfect BCC crystal bulk modulus of the empirical potential. Note that these high-dose simulations suggest that while the onset of the asymptotic microstructural state occurs at the dose close to $\phi \sim$ $2.5 \mathrm{cDPA}$, the ultimate steady state develops at a somewhat higher dose $\phi_{0} \sim 5 \mathrm{cDPA}$, in agreement with experimental observations $[33,38]$.

network. At $T=0 \mathrm{~K}$, the interstitial transport occurs via an athermal mechanism driven by the spatially fluctuating stress, where as at $300 \mathrm{~K}$ in pure iron some of the defects migrate via thermal activation, the latter resulting in increased recombination and thus a lower interstitial count. Despite the increased recombination rate, the increased mobility also makes it more likely that interstitials form clusters in a way that gives rise to a qualitatively similar behavior to the $0 \mathrm{~K}$ Frenkel pair insertion simulations.

Concluding this section, we note that while it is expected that thermally activated migration of defects would likely reduce the magnitude of swelling as well as the density of defects compared to predictions derived from a simulation describing the $T=0 \mathrm{~K}$ limit, in alloys or materials with complex microstructure, also containing a significant amount

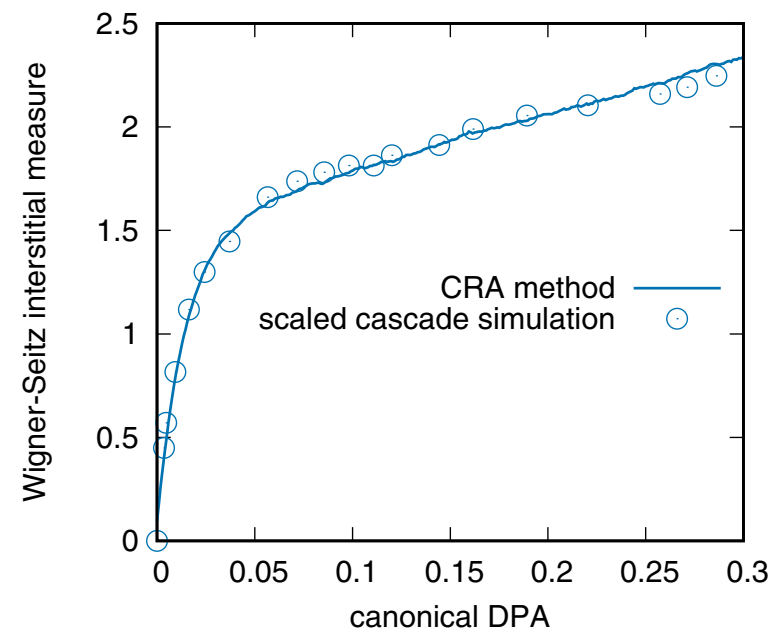

FIG. 13. Comparison of the interstitial defect content, in percent per lattice site units, versus canonical DPA derived from the Frenkel pair insertion method developed in this study, and the scaled data derived from cascade overlap simulations performed for pure $\mathrm{Fe}$ at $300 \mathrm{~K}$ in Ref. [81].

of impurities, the difference between predictions derived from low-temperature simulations and experimental observations might not be overly large. If the mobility of defects is impeded by their interaction with impurities, for example carbon and nitrogen interstitial atoms in steels, the defect immobilization effect would play a part equivalent to a significant reduction of temperature, making the results of this study pertinent to observations.

\section{LATTICE STRAIN AND SWELLING}

By plotting the evolving hydrostatic pressure divided by the appropriate bulk modulus, as is done in Fig. 2, we acquire an estimate for the volumetric swelling equivalent to our fixed volume FI simulations. To explore the quantities that can be observed experimentally to monitor the swelling of materials exposed to irradiation, we now relax the simulation cell corresponding to the resulting configurations while maintaining its orthorhombic symmetry. Such a relaxation involves a conjugate gradient relaxation of both the atomic coordinates and the simulation cell as a whole. A change in simulation cell volume will result in both an affine (homogeneous) and nonaffine (heterogeneous) displacement field of atomic coordinates. Inspection of the nonaffine component between the fixed-volume and resulting zero-stress configurations, reveals an average displacement of approximately $0.01 \AA$ and thus a negligible change in defect structure. This was confirmed by visual inspection of the unchanging defect structure.

Figure 14(a) plots the swelling (percentage volume increase) derived from the converged simulation cell volume as a function of cDPA. Inspection reveals that swelling is approximately $10 \%$ larger than the value predicted by the hydrostatic pressure to bulk modulus ratio, but otherwise it follows closely the evolution of the hydrostatic pressure derived from the fixed volume simulations illustrated in Fig. 2.

To investigate the evolution of the lattice strain developing as a result of accumulation of defects in the material, we calculate the $\mathrm{x}$-ray structure factor from which the location of 


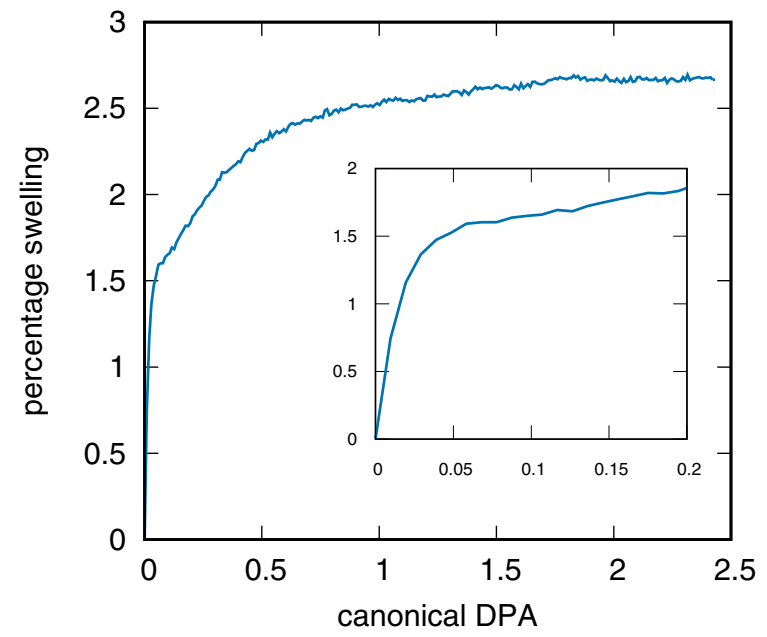

(a)

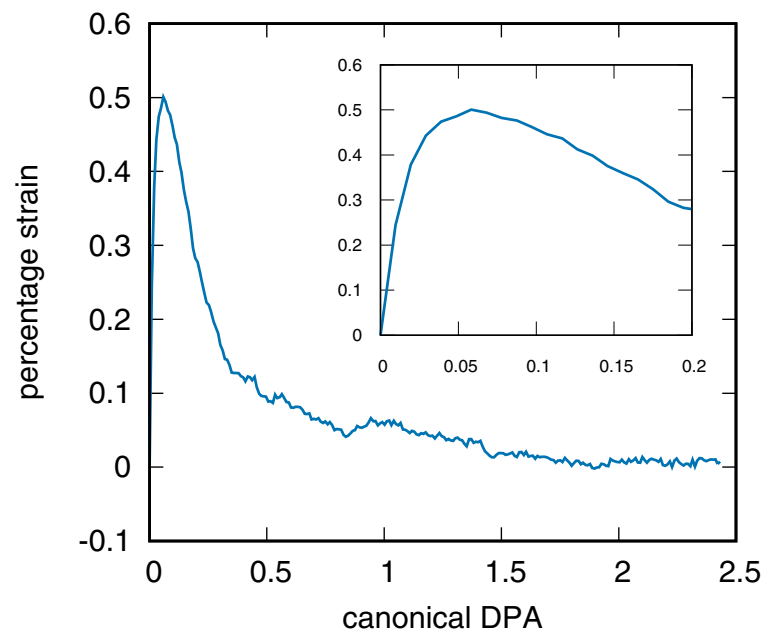

(b)

FIG. 14. (a) Percentage volume swelling derived from the orthorhombic cell relaxation performed at a fixed zero external stress. (b) Lattice strain derived from the 100 Bragg peak shift. In both figures, insets show a zoom-in of the low cDPA interval of microstructural evolution. In the limit of high dose, the lattice strain, primarily associated with the spatially localized isolated defects gradually diminishes whereas, the total swelling saturates at its highest value as a result of the formation of the system-spanning dislocation network.

the $\{100\}$ Bragg diffraction peak is determined as a function of cDPA. The examination of the $\mathrm{x}$-ray structure factor is a common way of experimental determination of strain $[82,83]$. In the present calculation of the structure factor, we do not include the scattering geometry or the appropriate atomic form-factor, which would be required for a direct comparison of predictions with observations, and simply calculate the magnitude squared of

$$
S(\mathbf{k})=\frac{1}{\sqrt{N}} \sum_{i} \exp \left(i \mathbf{k} \cdot \mathbf{r}_{i}\right),
$$

where $N$ is the number of atoms at positions $\mathbf{r}_{i}$ within the simulation box of volume $L_{x} L_{y} L_{z}$, and $\mathbf{k}=$ $2 \pi\left(n_{x} / L_{x}, n_{y} / L_{y}, n_{z} / L_{z}\right)$. Figure 15 shows two-dimensional cross-sections of scattered intensity generated using the atomic configurations corresponding to the cDPA values of 0.2 and 2.5 .

The corresponding lattice strain in the cell is determined from the position of the $\{100\}$ Bragg wave vector $k$ calculated via the relation $k_{0}\left(1 / k-1 / k_{0}\right)$, where $k_{0}$ is the $\{100\}$ Bragg wave vector corresponding to the initial ideal defect-free BCC lattice. Figure 14(b) plots this strain as a function of cDPA, with the inset showing its evolution at low cDPA values. An inspection of this figure demonstrates an initial increase of the lattice strain occurring during the linear FI insertion regime when each FI produces a vacancy and an isolated interstitial. The lattice strain reaches a maximum at a cDPA value of approximately $\phi=0.05$, which is precisely the point at which the interstitial defect content begins to decrease, signifying the beginning of the dislocation loop nucleation and growth regime. As the cDPA values continue to increase, the lattice strain decreases and nears zero in the limit when the microstructure has evolved into an asymptotic exposureinvariant steady state. A qualitatively similar trend has been seen in the X-ray derived strain for urania [58], although in that case, the final strain of the steady state was nonzero.

\section{DISCUSSION}

This work has explored how the microstructure of a metal evolves in response to the gradual accumulation of elementary radiation defects. The CRA algorithm, representing possibly the simplest conceivable model for the microstructural evolution of a material resulting from the accumulation of defects, provides a computationally expedient way of exploring the effect of irradiation on microstructure at atomic level resolution in the arbitrarily large dose limit. The analysis presented here focuses on the evolution occurring at low temperature, where the dynamics of microstructure is driven not by thermal activation but by the relaxation of spatially fluctuating stress generated by the defects. This mode of evolution, while often observed experimentally and encountered in applications, has so far presented the greatest difficulty to simulations.

The CRA model considered above has a unique feature that it presents a direct mapping of the dose to microstructure, treated in the statistical mechanics sense. In the presence of thermal activation, this unique mapping no longer exists, and microstructural evolution has to be treated in the space of three variables: time $t$, dose rate $\dot{\phi}=d \phi / d t$, and temperature $T$. In the limit $T=0$, microstructure is a unique function of dose $\phi=\int \dot{\phi} d t$.

By a direct simulation, we find that starting already from a relatively small dose $\phi \sim 0.02$ canonical DPA, microstructure evolves in a complex nonlinear manner, exhibiting a structural transition from an ensemble of isolated defects and clusters of defects to a steady-state microstructure dominated by a system-spanning dislocation network, resulting from the homogeneous nucleation and growth of interacting dislocation loops, embedded in a dense field of vacancies and vacancy clusters. The fact that vacancies and vacancy clusters, not detectable by conventional transmission electron microscopy, are present in irradiated materials at high concentration is well documented experimentally [77], and the simulations 
(a)

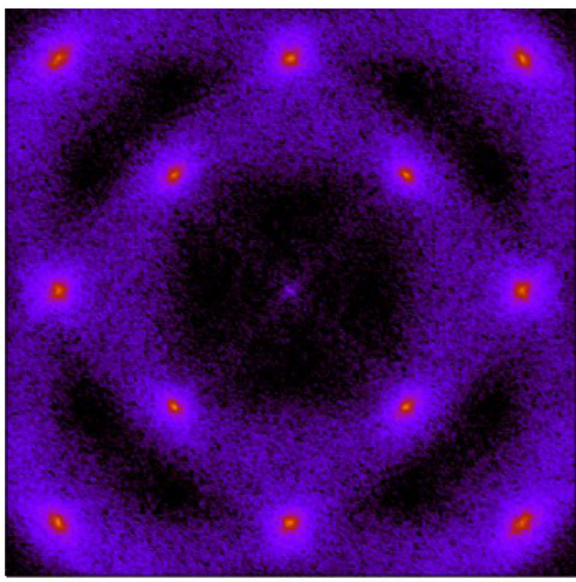

(b)

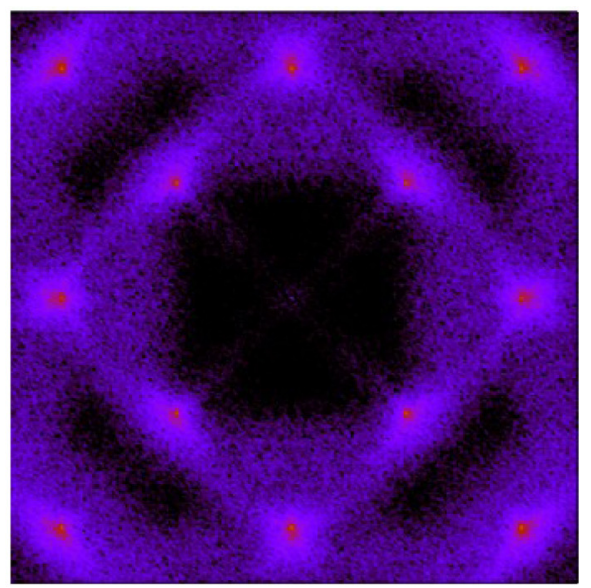

FIG. 15. Plots of $x$-ray scattering intensity computed for the $\{100\}$ family of Bragg peaks using atomic configurations simulated for the cDPA values of 0.2 and 2.5 . The intensity is shown using a logarithmic scale.

presented here show how such vacancy-rich microstructures form.

In Sec. IV, we see that the potential energy landscape of this steady state exhibits a hierarchy of energy and lengthscales that directly correspond to the evolving defect content driven purely by spatially fluctuating stress. In particular, it is variations in the dislocation configuration that affect the largest energy and length scales of this microstructural landscape through changes in the internal stress at the scale of nanometers and beyond. This is most strikingly displayed through Fig. 6, which plots the probability distribution for the change in energy due to a single Frenkel pair insertion, with a similar distribution found for the changes in stress. Whilst the total first moment of this distribution is zero, thus ensuring the steady-state condition, Fig. 6(b) demonstrates that a resulting drop in energy can range from the electron-volt energy scale associated with a single Frenkel pair annihilation, to hundreds of electron-volt energy scale due to dislocation reorganization. The statistics of this latter regime is characterized by a powerlaw tail with an exponent greater than three. This, distinctly non-Gaussian, part of the distribution reflects the strongly correlated nature of the resulting microstructure, where spatially separated regions cannot be considered independent from each other.

The large value of the exponent does however ensure a finite first and second moment for the associated drop in energy due to Frenkel pair insertion, and thus a well defined steady state. However, a diverging fourth (and higher) order moment suggests that strong deviations, whilst rare, will occur, corresponding to large changes in energy and dislocation network configuration. That such a strong material response can occur due to a local perturbation, indicates that the underlying steady-state microstructure has features which may be understood from the perspective of criticality [84]. Dislocation structures are known to exhibit regimes of spatial and/or temporal scale-invariance, described by various power-law distributions, which is characteristic of an out-ofequilibrium critical point $[85,86]$, and whose response to a global perturbation such as an external load may fall into a certain universality class that defines the critical state. Within this context, it will be desirable to investigate the emergent nonuniversal structural length scales of our steady state that ultimately would suppress such scale-free behavior and result in a statistically meaningful average material response: a highly desirable property of an engineering-relevant material.

As a function of dose, the evolution of miscrostructure is nonlinear and nonmonotonic. While the defect content and the resulting internal pressure vary monotonically as functions of dose, the microstructure itself evolves from a state characterized by the gradually increasing density of defects and clusters of defects to a state involving an extended large-scale dislocation network, forming as a result of interaction and coalescence of loops. This is expected to influence a range of properties, including the ease of recovery of defects that would require a significantly higher annealing temperature at a dose above $0.05 \mathrm{DPA}$ in comparison with the low-dose exposure; the likely effect on mechanical properties characterized for example by a nonmonotonic variation of radiation

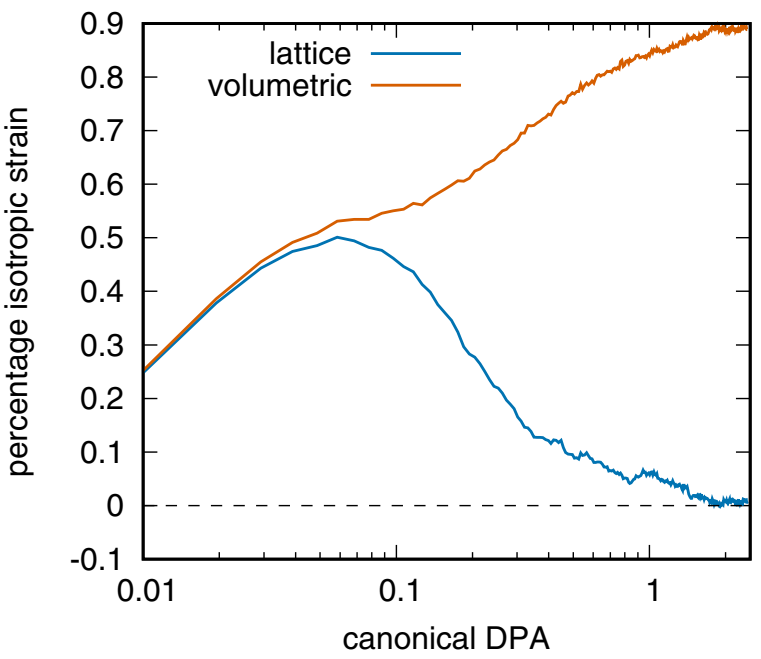

FIG. 16. Plots of isotropic volumetric strain obtained from swelling [Fig. 14(a)] and isotropic lattice strain obtained from x-ray diffraction data [Fig. 14(b)], and shown as a function of canonical DPA (Frenkel pair insertion number divided by the total number of atoms). 


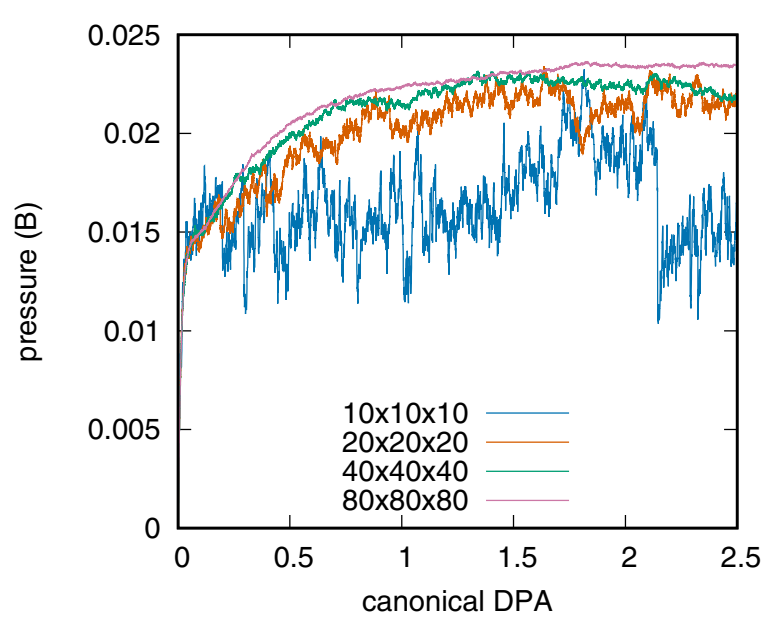

(a)

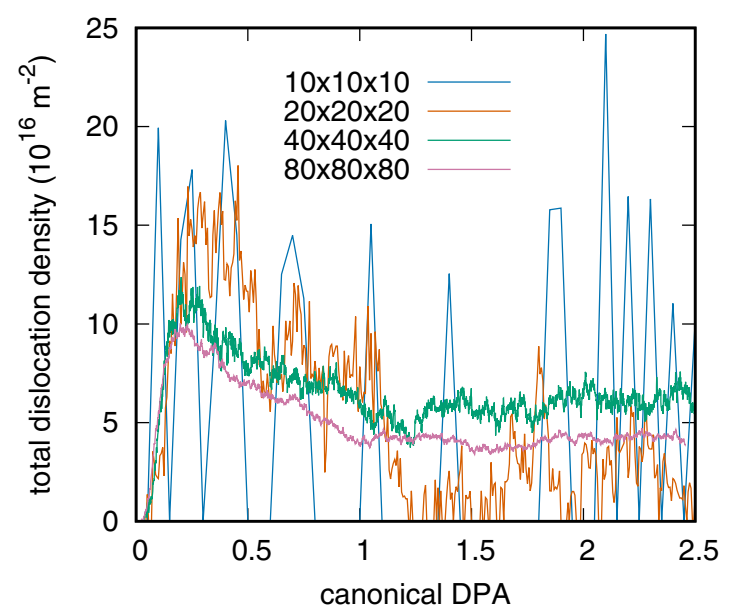

(b)

FIG. 17. (a) Global hydrostatic pressure and (b) total dislocation density as a function of canonical DPA (Frenkel pair insertion number divided by the total number of atoms) for BCC iron for four system sizes.

hardening as a function of dose, with a peak at $\sim 0.1 \mathrm{DPA}$ or below; a potentially significant role of climb in relation to mechanical deformation, as the dislocation network driven by the applied stress in a dense ensemble of defects would evolve differently from predictions based on an application of the Orowan model [87]; and possible implications for radiation embrittlement where a complex microstructure evolving in the high-dose limit cannot be adequately represented by a set of spatially well separated obstacles.

How do the above simulations compare with experimental observations? At the beginning of this study, we noted that the intrinsic relaxation timescales of evolution of microstructure may be macroscopic even if the temperature is relatively high, and can vary from hours [40,41] to weeks [42] and even months [38]. If the dose-rate is sufficiently high such that the corresponding irradiation timescale is shorter than the relaxation time of the evolving microstructure, athermal stress driven processes will dominate the evolution. This regime of response will always be the case at low enough temperatures, but can also be relevant at ambient or elevated temperatures if the relaxation time of the steady-state microstructure is sufficiently large. This view point represents a change in paradigm from the usual perspective that thermally activated processes are primarily responsible for microstructural evolution. The present work and the associated CRA algorithm offer a viable modeling strategy to investigate this new paradigm. Indeed, with minimal computational effort, a steady-state microstructure is achieved that is resistant to canonical irradiation and has strong qualitative similarities with what is seen experimentally in the high-dose limit. That such a microstructure can be produced purely through stress driven processes is remarkable and best demonstrated by comparing the volumetric strain, measured in terms of a bulk volume change, and lattice strain, measured in terms of lattice expansion, see Sec. VI. Figure 16 combines the data of Fig. 14 and shows the volumetric strain obtained by relaxing the simulation cell volume to a zero pressure state, and the average lattice strain, multiplied by three, obtained via a peak-shift analysis of $\mathrm{x}$-ray diffraction spectra simulated as a function of canonical DPA. The figure demonstrates good initial agreement between the two isotropic strain quantities at low cDPA, reflecting the fact that the origin of swelling in the dilute dose regime is due to the lattice strain of point defects. At a higher dose, the two strain measures diverge, with the lattice strain approaching zero in the steady-state limit. As already discussed in Sec. VI, this result demonstrates that the athermal stress-driven simulation protocol readily generates the extra full atomic planes that contribute to the swelling phenomenon.

Concluding this study, we note that the above analysis and numerical results have direct implications for modeling structural components on the macroscopic scale [78], and the computational methodology should help address the still outstanding challenges of nuclear engineering and reactor design.

\section{ACKNOWLEDGMENTS}

This work has been carried out within the framework of the EUROfusion Consortium and has received funding from the Euratom research and training programme 20142018 and 2019-2020 under Grant Agreement No. 633053. The views and opinions expressed herein do not necessarily reflect those of the European Commission. This work also received funding from the Euratom research and training programme 2019-2020 under Grant Agreement No. 755039. We acknowledge funding by the RCUK Energy Programme (Grant No. EP/T012250/1) and support from the EPFL Swiss Plasma Center. We are grateful for stimulating discussions with D. Perez, F. Hofmann, D. R. Mason, M. Boleininger, M.-C. Marinica, M.-J. Caturla, and P.-W. Ma, and would also like to thank R. A. Akers and S. de Witt for providing access to computing resources managed by the IRIS (STFC) Consortium.

\section{APPENDIX A: SYSTEM SIZE EFFECT}

Here we consider the effect of system size upon application of the CRA method through the study of those quantities shown in Sec. III for the case of the $80 \times 80 \times 80$ BCC unit 


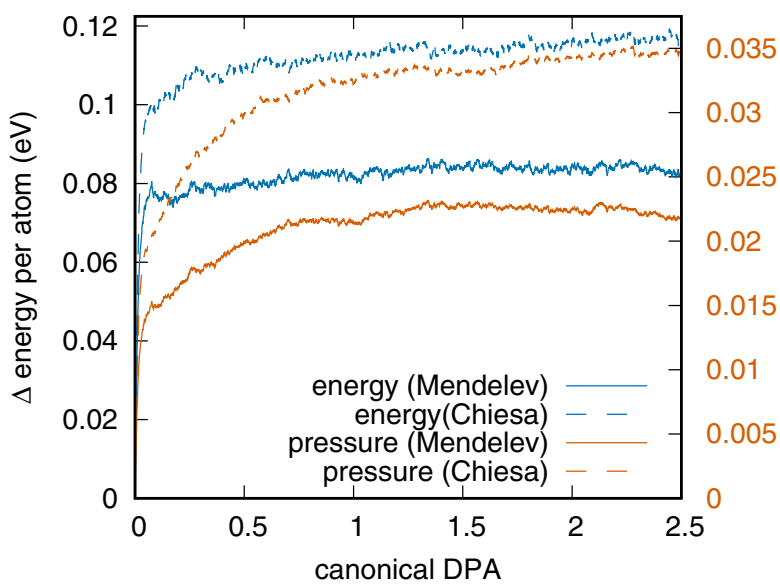

(a)

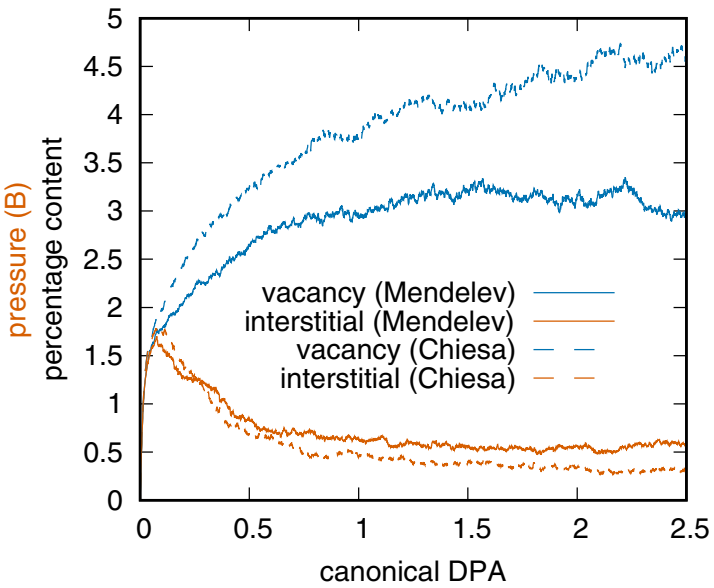

(b)

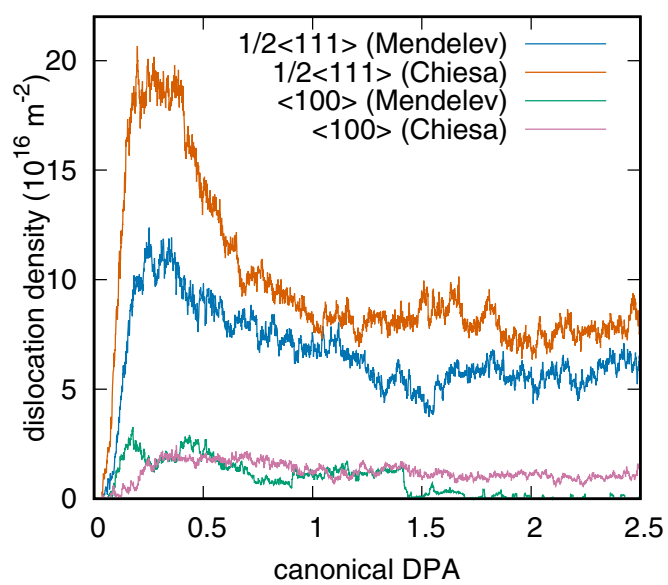

(c)

FIG. 18. (a) Change in energy per atom and hydrostatic pressure, (b) vacancy and lattice interstitial content, and (c) density of dislocations with Burgers vectors $1 / 2\langle 111\rangle$ and $\langle 100\rangle$ shown as a function of canonical DPA (Frenkel pair insertion number divided by the total number of atoms). Data are shown for the Fe empirical potentials of Mendelev et al. [66] and Chiesa et al. [67], for a system size of $40 \times 40 \times 40 \mathrm{BCC}$ unit cells.

cell. Here, three smaller simulation cell sizes are considered: $10 \times 10 \times 10,20 \times 20 \times 20$ and $40 \times 40 \times 40$ again using the Mendelev Fe potential. Figure 17(a) plots the evolution of the global hydrostatic pressure as a function of canonical DPA. For the smallest system size considered, the pressure is somewhat lower and more strongly fluctuating. As the system size increases, the magnitude of fluctuations diminishes and the steady-state pressure approaches that of the largest cell size of $80 \times 80 \times 80$. The change in energy per atom as a function of canonical DPA for different system sizes (not shown) shows a dependence that on average is independent of system size, however, with an increase in the magnitude of fluctuations as the system size decreases. The results of Secs. III and IV which demonstrated that strong local variations in internal stress correspond to reorganizations in the dislocation network structure, suggest that the increased fluctuations in both energy and pressure as the system size reduces originate from an increase in the fluctuations of the corresponding lattice interstitial and dislocation populations, where for the smallest system size small dislocation loops can nucleate, grow and disappear as they approach the size of the simulation cell-to be replaced by a system spanning extra plane of atoms. This is confirmed by inspection of the evolving dislocation content. Figure 17(b) plots the total dislocation content as a function of canonical DPA for the four system sizes considered, and it is seen that for the smallest system size of $10 \times 10 \times 10$ unit cells the dislocation content fluctuates between zero and a finite value larger than that of the $80 \times 80 \times 80 \mathrm{BCC}$ unit cell sample.

\section{APPENDIX B: COMPARISON OF DIFFERENT Fe POTENTIALS}

Here we consider the dependence of the response of the material to multiple Frenkel pair insertions on the choice of the $\mathrm{Fe}$ empirical potential describing the interaction between the atoms. The potentials we compare are the EAM potentials of Mendelev et al. [66] and the second generation magnetic potential of Chiesa et al. [67]. Figure 18 plots the change in energy per atom and hyrdrostatic pressure, vacancy and lattice 


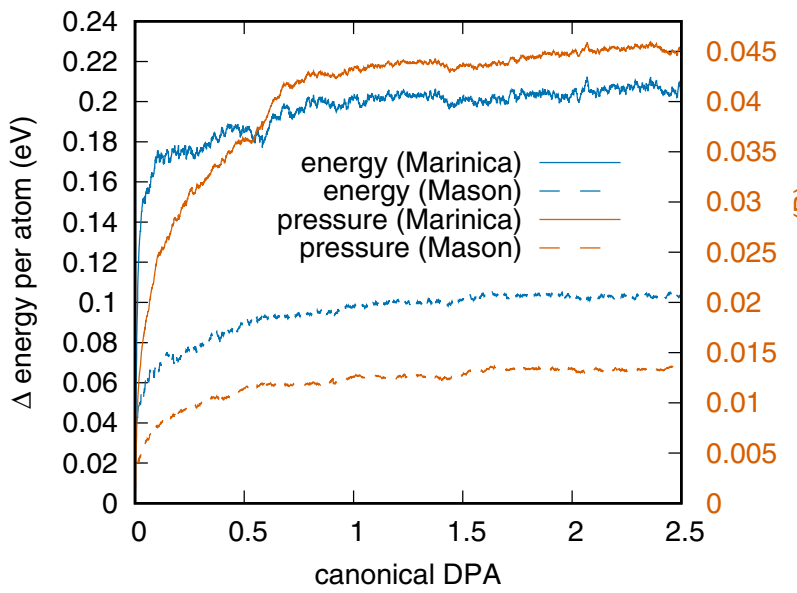

(a)

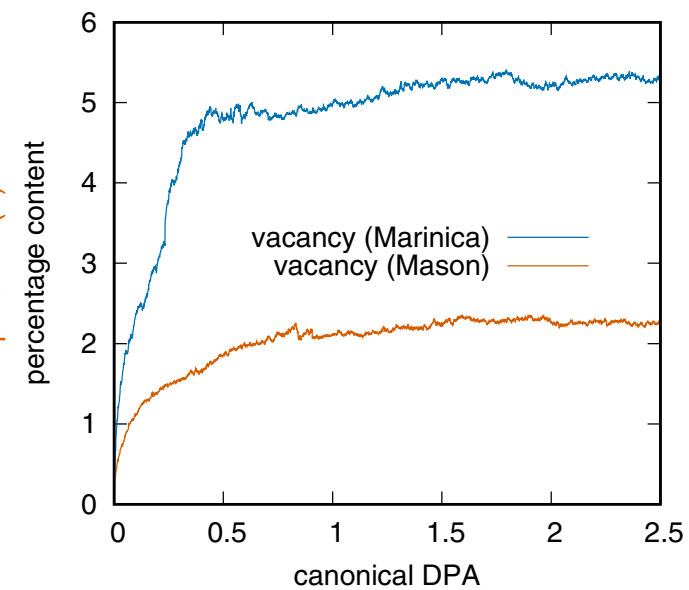

(b)

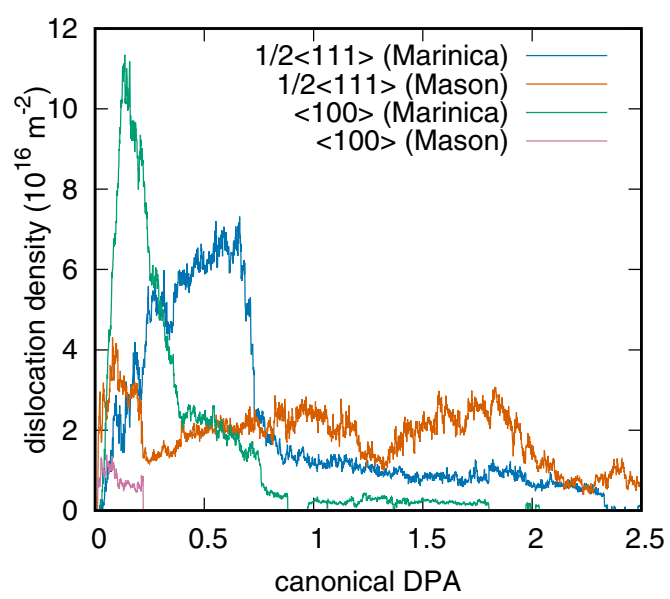

(c)

FIG. 19. (a) Change in energy per atom and hydrostatic pressure, (b) vacancy content, and (c) density of dislocations with Burgers vectors $1 / 2\langle 111\rangle$ and $\langle 100\rangle$ shown as a function of canonical DPA (Frenkel pair insertion number divided by the total number of atoms). Data are shown for the $\mathrm{W}$ empirical potentials of Marinica et al. [68] and Mason et al. [69] and the system size of $40 \times 40 \times 40 \mathrm{BCC}$ unit cells.

interstitial population and total dislocation line density as a function of canonical DPA for the system size of $40 \times 40 \times$ 40. Figure 18(a) demonstrates a qualitative similarity between the empirical potentials for the evolving change in energy and pressure. The Chiesa Fe magnetic potential is also reaching a steady state beyond the cDPA value of $\phi \sim 1$, however the final change in energy and pressure is non-negligibly larger than for the Mendelev Fe potential. Hence there are quantitative differences in the steady-state energy storage properties between the potentials. The origin of this non-negligible difference between the potentials, predicting nearly identical formation energies of defects, may be seen in the lattice and line defect populations shown in Figs. 18(b) and 18(c), where the vacancy and $1 / 2\langle 111\rangle$ dislocation populations are larger, whereas the lattice interstitial and $\langle 100\rangle$ dislocation populations are smaller for the Chiesa potential. Indeed, the ratio between the different defect concentrations of the two $\mathrm{Fe}$ potentials is comparable to the ratio of the differences between the energy and pressure seen in Fig. 18(a). The general evolution of these defect populations is however qualitatively the same, reflecting the empirical potential independence of the general picture of microstructural evolution involving a dilute lattice defect regime, followed by a interstitial loop nucleation and growth regime, and finally a loop coalescence regime as the material microstructure enters the steady-state regime.

\section{APPENDIX C: TUNGSTEN}

The Frenkel pair insertion method is now applied to BCC Tungsten using the potentials of Marinica et al. [68] and Mason et al. [69]. Figure 19 shows data similar to that shown for Fe in Fig. 18. Figure 19(a) demonstrates that both potentials for $\mathrm{W}$ exhibit similar behavior as Fe for the evolving change in energy and pressure. The two $\mathrm{W}$ potentials demonstrate quantitative differences between the steady-state values of pressure and energy. Figure 19(b) plots the evolving vacancy concentration with respect to canonical DPA. For the case of lattice interstitials, the approach used for distinguishing lattice interstitial defects from that of extra-half planes does not work for W. This is most likely due to the more delocalized nature of the $\langle 111\rangle$ crowdion interstitial defect when compared to that of the $\langle 110\rangle$ dumbbell interstitial geometry of Fe. Like that of Fe, the ratio of the different vacancy concentrations is comparable to the ratio of the different pressure and energies 
between the two potentials suggesting the differences seen in Fig. 19(a) might originate in the different defect concentrations of the potentials. This difference in defect densities is also seen in the different dislocation line densities shown in Fig. 19(c). Specific to W, Fig. 19(c) shows that for the Marinica potential, an initial dominance of small $\langle 100\rangle$ loops, whereas for the potential of Mason, $1 / 2\langle 111\rangle$ loops initially dominate. In the steady-state regime, both potentials exhibit the dominant presence of $1 / 2\langle 111\rangle$ loops. The origin of this difference lies in the incorrect ordering of $\langle 100\rangle$ and $1 / 2\langle 111\rangle$ loop energies, found in simulations that use the Marinica potential to model small loops [88].
[1] R. G. Alía, M. Brugger, S. Danzeca, F. Cerutti, J. P. de Carvalho Saraiva, R. Denz, A. Ferrari, L. L. Foro, P. Peronnard, K. Røed, R. Secondo, J. Steckert, Y. Thurel, I. Toccafondo, and S. Uznanski, Single event effects in high-energy accelerators, Semicond. Sci. Technol. 32, 034003 (2017).

[2] B. van der Schaaf, C. Petersen, Y. De Carlan, J. W. Rensman, E. Gaganidze, and X. Averty, High dose, up to $80 \mathrm{dpa}$, mechanical properties of Eurofer 97, J. Nucl. Mater. 386-388, 236 (2009).

[3] A. V. Krasheninnikov and F. Banhart, Engineering of nanostructured carbon materials with electron or ion beams, Nature (London) 6, 723 (2007).

[4] I. W. Donald, B. L. Metcalfe, and R. N. J. Taylor, The immobilization of high level radioactive wastes using ceramics and glasses, J. Mater. Sci. 32, 5851 (1997).

[5] J. R. Conrad, J. L. Radtke, R. A. Dodd, F. J. Worzala, and N. C. Tran, Plasma source ion-implantation technique for surface modification of materials, J. Appl. Phys. 62, 4591 (1987).

[6] F. A. Garner, M. B. Toloczko, and B. H. Sencer, Comparison of swelling and irradiation creep behavior of fcc-austenitic and bcc-ferritic/martensitic alloys at high neutron exposure, J. Nucl. Mater. 276, 123 (2000).

[7] K. Nordlund, S. J. Zinkle, A. E. Sand, F. Granberg, R. S. Averback, R. Stoller, T. Suzudo, L. Malerba, F. Banhart, W. J. Weber, F. Willaime, S. L. Dudarev, and D. Simeone, Improving atomic displacement and replacement calculations with physically realistic damage models, Nat. Commun. 9, 1084 (2018).

[8] G. H. Kinchin and R. S. Pease, The displacement of atoms in solids by radiation, Rep. Prog. Phys. 18, 1 (1955).

[9] M. J. Norgett, M. T. Robinson, and I.M. Torrens, A proposed method of calculating displacement dose rates, Nucl. Eng. Des. 33, 50 (1975).

[10] G. S. Was, Z. Jiao, E. Getto, K. Sun, A. M. Monterrosa, S. A. maloy, O. Anderoglu, B. H. Senser, and M. Hackett, Emulation of reactor irradiation damage using ion beams, Scr. Mater. 88, 33 (2014).

[11] S. J. Zinkle and L. L. Snead, Opportunities and limitations for ion beams in radiation effects studies: Bridging critical gaps between charged particle and neutron irradiations, Scr. Mater. 143, 154 (2017).

[12] D. S. Gelles, Microstructural examination of commercial ferritic alloys at 200 dpa, J. Nucl. Mater. 233-237, 293 (1996).

[13] T. Diaz de la Rubia and M. W. Guinan, New Mechanism of Defect Production in Metals: A Molecular-Dynamics Study of Interstitial-Dislocation-Loop Formation in High-Energy Displacement Cascades, Phys. Rev. Lett. 66, 2766 (1991).

[14] J.-M. Delaye, S. Peuget, G. Bureau, and G. Calas, Molecular dynamics simulation of radiation damage in glasses, J. NonCrys. Solids 357, 2763 (2011).

[15] F. Granberg, K. Nordlund, M. W. Ullah, K. Jin, C. Lu, H. Bei, L. M. Wang, F. Djurabekova, W. J. Weber, and Y. Zhang,
Mechanism of Radiation Damage Reduction in Equiatomic Multicomponent Single Phase Alloys, Phys. Rev. Lett. 116, 135504 (2016).

[16] K. Nordlund, S. J. Zinkle, A. E. Sand, F. Granberg, R. S. Averback, R. Stoller, T. Suzudo, L. Malerba, F. Banhart, W. J. Weber, F. Willaime, S. L. Dudarev and D. Simeone, Primary radiation damage: A review of current understanding and models, J. Nucl. Mater. 512, 450 (2018).

[17] A. F. Calder, D. J. Bacon, A. V. Barashev, and Y. N. Osetsky, On the origin of large interstitial clusters in displacement cascades, Philos. Mag. 90, 863 (2010).

[18] A. E. Sand, S. L. Dudarev and K. Nordlund, High-energy collision cascades in tungsten: Dislocation loops structure and clustering scaling laws, Europhys. Lett. 103, 46003 (2013).

[19] A. E. Sand, M. J. Aliaga, M. J. Caturla, and K. Nordlund, Surface effects and statistical laws of defects in primary radiation damage: Tungsten vs. iron, Europhys. Lett. 115, 36001 (2016).

[20] M. J. Aliaga, I. Dopico, I. Martin-Bragado, and M. J. Caturla, Influence of free surfaces on microstructure evolution of radiation damage in Fe from molecular dynamics and object kinetic Monte Carlo calculations, Phys. Status Solidi A 213, 2969 (2016).

[21] M. Samaras, P. M. Derlet, H. Van Swygenhoven, and M. Victoria, Computer Simulation of Displacement Cascades in Nanocrystalline Ni, Phys. Rev. Lett. 88, 125505 (2002).

[22] M. Samaras, P. M. Derlet, H. Van Swygenhoven, and M. Victoria, Radiation damage near grain boundaries, Philos. Mag. 83, 3599 (2003).

[23] S. Z. Xu, Z. M. Hao, Y. Q. Su, W. J. Hu, Y. Yu, and Q. Wan, Atomic collision cascades on void evolution in vanadium, Radiat. Eff. Def. Sol. 167, 12 (2012).

[24] F. Granberg, J. Byggmästar, and K. Nordlund, Cascade overlap with vacancy-type defects in Fe, Eur. Phys. J. B 92, 146 (2019).

[25] B. Q. Fu, S. P. Fitzgerald, Q. Hou, J. Wang, and M. Li, Effect of collision cascades on dislocations in tungsten: A molecular dynamics study, Nucl. Instrum. Methods Phys. Res., Sect. B 393, 169 (2017).

[26] H. Wang, J.-T. Tian, W. Zhou, X.-F. Chen, B. Bai, and J.-M. Xue, Collision cascades interact with an edge dislocation in bcc Fe: A molecular dynamics study, RSC Adv. 8, 14017 (2018).

[27] J. Byggmästar, F. Granberg, A. E. Sand, A. Pirttikoski, R. Alexander, M.-C. Marinica, and K. Nordlund, Collision cascades overlapping with self-interstitial defect clusters in Fe and W, J. Phys.: Condens. Matter 31, 245402 (2019).

[28] X.-M. Bai, A. F. Voter, R. G. Hoagland, M. Nastasi, and B. P. Uberuaga, Efficient annealing of radiation damage near grain boundaries via interstitial emission, Science 327, 1631 (2010). 
[29] J. B. Wallace, L. B. Bayu Aji, L. Shao, and S. O. Kucheyev, Impact of pre-existing disorder on radiation defect dynamics in Si, Sci. Rep. 9, 12377 (2019).

[30] F. Granberg, F. Djurabekova, E. Levo, and K. Nordlund, Damage buildup and edge dislocation mobility in equiatomic multicomponent alloys, Nucl. Instrum. Methods Phys. Res., Sect. B 393, 114 (2017).

[31] O. El-Atwani, N. Li, M. Li, A. Devaraj, J. K. S. Baldwin, M. M. Schneider, D. Sobieraj, J. S. Wróbel, D. Nguyen-Manh, S. A. Maloy, and E. Martinez, Outstanding radiation resistance of tungsten-based high-entropy alloys, Sci. Adv. 5, eaav2002 (2019).

[32] A. E. Sand, J. Byggmästar, A. Zitting, and K. Nordlund, Defect structures and statistics in overlapping cascade damage in fusion-relevant bcc metals, J. Nucl. Mater. 511, 64 (2018).

[33] E. Gaganidze, C. Petersen, E. Materna-Morris, C. Dethloff, O. J. Weiß, J. Aktaa, A. Povstyanko, A. Fedoseev, O. Makarov, and V. Prokhorov, Mechanical properties and TEM examination of RAFM steels irradiated up to 70 dpa in BOR-60, J. Nucl. Mater. 417, 93 (2011).

[34] A. E. Sand, J. Dequeker, C. S. Becquart, C. Domain, and K. Nordlund, Non-equilibrium properties of interatomic potentials in cascade simulations in tungsten, J. Nucl. Mater. 470, 119 (2016).

[35] C. P. Race, D. R. Mason, M. W. Finnis, W. M. C. Foulkes, A. P. Horsfield, and A. P. Sutton, The treatment of electronic excitations in atomistic models of radiation damage in metals, Rep. Prog. Phys. 73, 116501 (2010).

[36] T. D. Swinburne, P.-W. Ma, and S. L. Dudarev, Low temperature diffusivity of self-interstitial defects in tungsten, New J. Phys. 19, 073024 (2017).

[37] A. R. Allnatt and A. B. Lidiard, Atomic Transport in Solids, (Cambridge University Press, Cambridge, England, 1993), p. 5.

[38] K. Papamihail, K. Mergia, F. Ott, Y. Serruys, Th. Speliotis, G. Apostolopoulos, and S. Messoloras, Magnetic effects induced by self-ion irradiation of Fe films, Phys. Rev. B 93, 100404 (2016).

[39] J. Silcox and M. J. Whelan, Direct observations of the annealing of prismatic dislocation loops and of climb of dislocations in quenched aluminium, Philos. Mag. 5, 1 (1960).

[40] F. Ferroni, X. Yi, K. Arakawa, S. P. Fitzgerald, P. D. Edmondson, and S. G. Roberts, High temperature annealing of ion irradiated tungsten, Acta Mater. 90, 380 (2015).

[41] M. Topping, T. Ungár, C. P. Race, A. Harte, A. Garner, F. Baxter, S. Dumbill, P. Frankel, and M. Preuss, Investigating the thermal stability of irradiation-induced damage in a zirconium alloy with novel in situ techniques, Acta Mater. 145, 255 (2018).

[42] B. A. Gurovich, E. A. Kuleshova, D. A. Maltsev, Y. M. Semchenkov, A. S. Frolov, Y. I. Shtrombakh, and A. V. Shutikov, Mechanism of change in VVER-440, -1000 vessel material properties in irradiation-recovery annealing irradiation cycle, Atomic Energy 125, 95 (2018).

[43] C. Domain, C. S. Becquart, and L. Malerba, Simulation of radiation damage in Fe alloys: An object kinetic Monte Carlo approach, J. Nucl. Mater. 335, 121 (2004).

[44] M. J. Caturla, Object kinetic Monte Carlo methods applied to modeling radiation effects in materials, Comput. Mater. Sci. 156, 452 (2019).
[45] C. C. Fu, J. Dalla Torre, F. Willaime, J.-L. Bocquet, and A. Barbu, Multiscale modeling of defect kinetics in irradiated iron, Nat. Mater. 4, 68 (2005).

[46] C. J. Ortiz and M. J. Caturla, Cascade damage evolution: Rate theory versus kinetic Monte Carlo simulations, J. Comput.Aided Mater. Des. 14, 171 (2007).

[47] C. S. Becquart, C. Domain, U. Sarkar, A. De Backer, and M. Hou, Microstructural evolution of irradiated tungsten: Ab initio parametrization of an OKMC model, J. Nucl. Mater. 403, 75 (2010).

[48] T. S. Hudson, S. L. Dudarev, M. J. Caturla, and A. P. Sutton, Effects of elastic interactions on post-cascade radiation damage evolution in kinetic Monte Carlo simulations, Philos. Mag. 85, 661 (2005).

[49] E. Martinez, J. Marian, M. H. Kalos, and J. M. Perlado, Synchronous parallel kinetic Monte Carlo for continuum diffusionreaction systems, J. Comput. Phys. 227, 3804 (2008).

[50] D. R. Mason, X. Yi, M. A. Kirk, and S. L. Dudarev, Elastic trapping of dislocation loops in cascades in ion-irradiated tungsten foils, J. Phys.: Condens. Matter 26, 375701 (2014).

[51] L. Casillas-Trujillo, A. S. Ervin, L. B. Xu, A. Barashev, and $\mathrm{H} . \mathrm{X} . \mathrm{Xu}$, Dynamics of interaction between dislocations and point defects in bcc iron, Phys. Rev. Mater. 2, 103604 (2018).

[52] D. R. Mason, A. E. Sand, and S. L. Dudarev, Atomistic-object kinetic Monte Carlo simulations of irradiation damage in tungsten, Modell. Simul. Mater. Sci. Eng. 27, 055003 (2019).

[53] L. Koch, F. Granberg, T. Brink, D. Utt, K. Albe, F. Djurabekova, and K. Nordlund, Local segregation versus irradiation effects in high-entropy alloys: Steady-state conditions in a driven system, J. Appl. Phys. 122, 105106 (2017).

[54] R. E. Stoller, Primary radiation damage, in Comprehensive Nuclear Materials, edited by R. J. M. Konings (Elsevier, Dordrecht, The Netherlands, 2012), Vol. 1, pp. 293-332.

[55] D. L. Turcotte, Self-organized criticality, Rep. Prog. Phys. 62, 1377 (1999).

[56] A. Chartier and M.-C. Marinica, Rearrangement of interstitial defects in alpha-Fe under extreme condition, Acta Mater. 180, 141 (2019).

[57] C. Maxwell, J. Pencer, and E. Torres, Atomistic simulation study of clustering and evolution of irradiation-induced defects in zirconium, J. Nucl. Mater. 531, 151979 (2020).

[58] A. Chartier, C. Onofri, L. Van Brutzel, C. Sabathier, O. Dorosh, and J. Jagielski, Early stages of irradiation induced dislocations in urania, Appl. Phys. Lett. 109, 181902 (2016).

[59] E. Y. Chen, C. Deo, and R. Dingreville, Irradiation resistance of nanostructured interfaces in $\mathrm{Zr}-\mathrm{Nb}$ metallic multilayers, J. Mater. Res. 34, 2239 (2019).

[60] M. J. Sabochick and N. Q. Lam, Radiation-induced amorphization of ordered intermetallic compounds $\mathrm{CuTi}, \mathrm{CuTi}_{2}$, and $\mathrm{Cu}_{4} \mathrm{Ti}_{3}$ : A molecular dynamics study, Phys. Rev. B 43, 5243 (1991).

[61] Y. Limoge, A. Rahman, H. Hsieh, and S. Yip, Computer simulation studies of radiation induced amorphization, J. Noncrystalline Solids 99, 75 (1988).

[62] D. S. Aidhy, P. C. Millett, T. Desai, D. Wolf, and S. R. Phillpot, Kinetically evolving irradiation-induced point defect clusters in $\mathrm{UO}_{2}$ by molecular dynamics simulation, Phys. Rev. B 80, 104107 (2009). 
[63] C. Jiang, M.-J. Zheng, D. Morgan, and I. Szlufarska, Amorphization Driven by Defect-Induced Mechanical Instability, Phys. Rev. Lett. 111, 155501 (2013).

[64] C. Liu, Y. Li, T. Shi, Q. Peng, and F. Gao, Oxygen defects stabilize the crystal structure of $\mathrm{MgAl}_{2} \mathrm{O}_{4}$ spinel under irradiation, J. Nucl. Mater. 527, 151830 (2019).

[65] X. Yi, A. E. Sand, D. R. Mason, M. A. Kirk, S. G. Roberts, K. Nordlund, and S. L. Dudarev, Direct observation of size scaling and elastic interaction between nano-scale defects in collision cascades, Europhys. Lett. 110, 36001 (2015).

[66] M. I. Mendelev, S. Han, D. J. Srolovitz, G. J. Ackland, D. Y. Sun, and M. Asta, Development of new interatomic potentials appropriate for crystalline and liquid iron, Philos. Mag. 83, 3977 (2003).

[67] S. Chiesa, P. M. Derlet, S. L. Dudarev, and H. Van Swygenhoven, Optimization of the magnetic potential for $\alpha$-Fe, J. Phys.: Condens. Matter 23, 206001 (2011).

[68] M.-C. Marinica, L. Ventelon, M. R. Gilbert, L. Proville, S. L. Dudarev, J. Marian, G. Bencteux, and F. Willaime, Interatomic potentials for modeling radiation defects and dislocations in tungsten, J. Phys.: Condens. Matter 25, 395502 (2013).

[69] D. R. Mason, D. Nguyen-Manh, and C. S. Becquart, An empirical potential for simulating vacancy clusters in tungsten, J. Phys.: Condens. Matter 29, 505501 (2017).

[70] S. Plimpton, Fast parallel algorithms for short-range molecular dynamics, J. Comput. Phys. 117, 1 (1995).

[71] A. Stukowski, Visualization and analysis of atomistic simulation data with OVITO-the Open Visualization Tool, Modell. Simul. Mater. Sci. Eng. 18, 015012 (2010).

[72] C. Björkas, K. Nordlund, and M. J. Caturla, Influence of the picosecond defect distribution on damage accumulation in irradiated $\alpha$-Fe, Phys. Rev. B 85, 024105 (2012).

[73] S. L. Dudarev, M. R. Gilbert, K. Arakawa, H. Mori, Z. Yao, M. L. Jenkins, and P. M. Derlet, Langevin model for real-time Brownian dynamics of interacting nanodefects in irradiated metals, Phys. Rev. B 81, 224107 (2010).

[74] S. L. Dudarev, K. Arakawa, X. Yi, Z. Yao, M. L. Jenkins, M. R. Gilbert, and P. M. Derlet, Spatial ordering of nano-dislocation loops in ion-irradiated materials, J. Nucl. Mater. 455, 16 (2014).

[75] A. Stukowski and K. Albe, Extracting dislocations and nondislocation crystal defects from atomistic simulation data, Modell. Simul. Mater. Sci. Eng. 18, 085001 (2010).
[76] A. Stukowski, V. V. Bulatov, and A. Arsenlis, Automated identification and indexing of dislocations in crystal interfaces, Modell. Simul. Mater. Sci. Eng. 20, 085007 (2012).

[77] E. Meslin, M. Lambrecht, M. Hernández-Mayoral, F. Bergner, L. Malerba, P. Pareige, B. Radiguet, A. Barbu, D. GómezBriceño, A. Ulbricht, and A. Almazouzi, Characterization of neutron-irradiated ferritic model alloys and a RPV steel from combined APT, SANS, TEM and PAS analyses, J. Nucl. Mater. 406, 74 (2010).

[78] S. L. Dudarev, D. R. Mason, E. Tarleton, P.-W. Ma, and A. E. Sand, A multi-scale model for stresses, strains and swelling of reactor components under irradiation, Nucl. Fusion 58, 126002 (2018).

[79] Z. S. Basinski, M. S. Duesbery, and R. Taylor, Influence of shear stress on screw dislocations in a model sodium lattice, Can. J. Phys. 49, 2160 (1971).

[80] R. von Mises, Mechanik der festen Körper im plastischdeformablen Zustand, Nachrichten von der Gesellschaft der Wissenschaften zu Göttingen, Mathematisch-Physikalische Klasse 1, 582 (1913).

[81] J. Byggmästar, F. Granberg, and K. Nordlund, Effects of the short-range repulsive potential on cascade damage in Iron, J. Nucl. Mater. 508, 530 (2018).

[82] F. Hofmann, D. Nguyen-Manh, M. R. Gilbert, C. E. Beck, J. K. Eliason, A. A. Maznev, W. Liu, D. E. J. Armstrong, K. A. Nelson, and S. L. Dudarev, Lattice swelling and modulus change in a helium-implanted tungsten alloy: X-ray microdiffraction, surface acoustic wave measurements, and multiscale modelling, Acta Mater. 89, 352 (2015).

[83] F. Hofmann, E. Tarleton, R. J. Harder, N. W. Phillips, P.-W. Ma, J. N. Clark, I. K. Robinson, B. Abbey, W. Liu, and C. E. Beck, 3D lattice distortions and defect structures in ion-implanted nano-crystals, Sci. Rep. 7, 45993 (2017).

[84] J. P. Sethna, K. A. Dahmen, and C. R. Myers, Crackling noise, Nature (London) 410, 242 (2001).

[85] M. Zaiser, Scale invariance in plastic flow of crystalline solids, Adv. Phys. 55, 185 (2006).

[86] R. Maass and P. M. Derlet, Micro-plasticity and recent insights from intermittent and small-scale plasticity, Acta Mater. 143, 338 (2018).

[87] D. J. Bacon and Y. N. Osetsky, Mechanisms of hardening due to copper precipitates in $\alpha$-iron, Philos. Mag. 89, 3333 (2009).

[88] J. Fikar and R. Gröger, Shape of small prismatic dislocation loops in tungsten and iron, Solid St. Phen. 258, 97 (2016). 